\title{
A New Theoretical Technique for the Measurement of High-Frequency Relic Gravitational Waves
}

\author{
R. Clive Woods ${ }^{1}$, Robert M. L. Baker ${ }^{2}$, Fangyu Li $^{3}$, Gary V. Stephenson ${ }^{4}$, \\ Eric W. Davis ${ }^{5}$, Andrew W. Beckwith ${ }^{2,3}$ \\ ${ }^{1}$ Department of Electrical and Computer Engineering, Louisiana State University, \\ Baton Rouge, USA \\ ${ }^{2}$ GravWave ${ }^{\circledR}$ LLC and Transportation Sciences Corporation, \\ Playa del Rey, USA \\ ${ }^{3}$ Department of Physics, Chongqing University, Chongqing, \\ People's Republic of China \\ ${ }^{4}$ Seculine Consulting, Redondo Beach, USA \\ ${ }^{5}$ Institute for Advanced Studies at Austin, Austin, USA \\ E-mail:DrRobertBaker@GravWave.com \\ Received March 3, 2011; revised April 17, 2011; accepted April 27, 2011
}

\begin{abstract}
Under most models of the early universe evolution, high-frequency gravitational waves (HFGWs) were produced. They are referred to as "relic" high-frequency gravitational waves or HFRGWs and their detection and measurement could provide important information on the origin and development of our Universe-information that could not otherwise be obtained. So far three instruments have been built to detect and measure HFRGWs, but so far none of them has achieved the required sensitivity. This paper concerns another detector, originally proposed by Baker in 2000 and patented, which is based upon a recently discovered physical effect (the Li effect); this detector has accordingly been named the "Li-Baker detector". The detector has been a joint development effort by the P. R. China and the United States HFGW research teams. A rigorous examination of the detector's performance is important in the ongoing debate over the value of attempting to construct a Li-Baker detector and, in particular, an accurate prediction of its sensitivity in the presence of significant noise will decide whether the Li-Baker detector will be capable of detecting and measuring HFRGWs. The potential for useful HFRGW measurement is theoretically confirmed.
\end{abstract}

Keywords: High-Frequency Gravitational Waves, High-Frequency Relic Gravitational Wave Measurement, Primordial Gravitational Waves, Microwaves, Cosmology, General Relativity

\section{Introduction}

Thus far three instruments have been built to detect and measure high-frequency gravitational waves or HFGWs [1-6], but so far none of them has achieved the required sensitivity to detect and measure "relic" HFGWs from the early universe or HFRGWs. This paper concerns another detector, originally proposed by Baker in 2000 and patented [7], which is based upon a recently discovered physical effect by Li [8]; this detector has accordingly been named the "Li-Baker detector." The detector has been a joint development effort by the P. R. China and the United States HFGW research teams.

Subsequent to the 2008 publication in EPJC, it has been generally accepted that a perturbative photon flux (PPF) is generated by high-frequency gravitational waves (HFGWs) in the presence of a static magnetic field and an electromagnetic (EM) beam having the same frequency and a suitable phase difference (synchroresonance) superimposed on the HFGW [9]. This effect is discussed in Section 3 and its proof summarized in the Appendix. In order to utilize this new theoretical technique for detection of cosmologically generated HFRGWs two important questions arise: 1) What are the characteristics and cosmological significance of the relic highfrequency gravitational waves from the early universe and 2) what is the adverse effect of the noise generated by the relatively intense EM beam on the ability to detect 
and measure the very weak PPF signal that they produce? An objective of this paper is to answer these questions.

\section{High-Frequency Relic Gravitational Waves}

\subsection{Cosmological Significance of the Relic High-Frequency Gravitational Waves from the Early Universe}

Although there is no definite evidence of the existence of HFRGWs their measurement would allow for validation or falsification of various cosmological theories. Most models of early universe evolution predict that HFGWs were produced as a result of the violent expansion of the young universe. and their measurement and characterization could provide important information on the origin and development of our Universe since their properties were uniquely determined by the most violent event in the history of the Universe. This information is a vital piece in the jigsaw of understanding how the young universe evolved, and that information cannot be obtained by any other means. A number of techniques have been proposed for measuring relic GWs at both low frequency and high frequency, and some GW detectors have been built, so far without any success in detection. Like the Laser Interferometer Space Antenna (LISA) [10], the swarm of Cosmic Microwave Background (CMB) sensors [11] and the Russian gravitational-electromagnetic resonance high-frequency gravitational wave detector $[12,13]$ (all proposed for sensing primordial or relic gravitational waves), no Li-Baker detector has yet been constructed.

As is well known, Einstein [14] predicted the possibility of waves in four-dimensional spacetime, i.e., the usual three dimensions of space plus time. These waves are gravitational waves whose spacetime strain is $h$. This spacetime strain is analogous to mechanical strain in a beam, and is the ratio of the change in length to the original length (without the stress of a passing gravitational wave). Thus, the strain, $h$, has units of meters per meter $(\mathrm{m} / \mathrm{m})$ and is dimensionless as is its amplitude $A$.

The importance of measuring the HFRGW strain $h$ and dimensionless energy density $\Omega_{g w}$ is that predictions of their values produced by the "Big Bang" under inflationary universe models [15-20] and cosmological string scenarios [21-23] are available, and so direct measurement will allow discrimination between the various models. Many of these models predict maximum HFRGW amplitude around $10 \mathrm{GHz}$, with $h$ in the approximate range $10^{-30}$ to $\sim 10^{-34}$. Low-frequency gravitational wave detectors such as LIGO, based upon optical interferometers, have an optimal detection frequency $\sim 100 \mathrm{~Hz}$ with upper frequency detection limit of $\sim 2000$ $\mathrm{Hz}$, and accordingly cannot detect HFRGWs [24]. In order to detect and measure high frequencies at small amplitudes, detectors utilizing different techniques must be employed, complementary to the low frequency detectors. Krauss, Scott and Meyer [11] suggest “... primordial (relic) gravitational waves also leave indirect signatures that might show up in CMB (Cosmic Wave Background) maps." They propose the use of thousands of new detectors (possibly as many as 50,000) as well as spacecraft-borne detectors to obtain the required sensitivity.

Theorized cosmological signatures (i.e., frequency spread, polarization and phase) of the HFRGWs are important because of the uncertainty surrounding cosmological parameters leading to variations in the early universe [25]. One of the most important parameters for analysis of the beginning of the Universe is the dimensionless relic gravitational wave energy density, $\Omega_{g w}[19$, 26-28]. According to these estimates, the upper limit of $\Omega_{g w}$ for relic GWs should be smaller than $10^{-5}$. In fact, recent estimates [10] show that the upper limit of $\Omega_{g w}$ should be $6.9 \times 10^{-6}$ at about $100 \mathrm{~Hz}$. The spectra of dimensionless primordial relic GW strains $h$ as a function of frequency have been estimated in detail by Grishchuk [19,26-28]. Detailed observational data for $h$ and its variation in time and direction can be used to refine the estimated value of $\Omega_{g w}$, and hence to differentiate among the competing cosmological theories for the beginning of the Universe.

\subsection{Presently Fabricated and Proposed HFGW Detectors}

As has been mentioned three high-frequency gravitational wave (HFGW) detectors have been built [29] and another has been proposed [12,13], all utilizing different measurement techniques. These are promising for future detection of HFRGWs having frequencies above 100 $\mathrm{kHz}$ (the definition of HFGWs adopted by Douglass and Braginsky [30]), but their sensitivities are each many orders of magnitude less than that required to detect and measure primordial HFRGWs. These existing HFGW detectors deal with the detection of a single plane HFRGW, but a stochastic background needs at least two detectors to be utilized for cross correlating the observed data. It is intended, therefore, that the Li-Baker detectors be utilized in pairs, which would also guard against false alarms. The Li-Baker detector is direction dependent and a background can be seen as a stochastic superposition of many plane waves propagating in all directions. The directionality is discussed in Section 4.4.

The first of these HFGW detectors has been con- 
structed at Birmingham University, England. The Birmingham HFGW detector measures changes in the polarization state of a microwave beam (indicating the presence of a $\mathrm{GW}$ ) moving in a waveguide $[1,2]$. It is expected to be sensitive to HFRGWs having spacetime strains of $h \sim 2 \times 10^{-13}$.

The second detector, built at INFN Genoa, Italy. It is a resonant HFRGW detector, comprising two coupled, superconducting, spherical, resonant chambers a few centimeters in diameter and configured as oscillators. The oscillators are designed to have (when uncoupled) almost equal resonant frequencies and when the frequency of the HFGW is just equal to the frequency difference between the normal modes in the two coupled spherical cavities, the EM energy conversion between the cavities will be maximum and the HFGW sensed. The system is expected to have a sensitivity to HFRGWs of about $h \sim 2 \times 10^{-17}$ with future expectation of $\sim 3 \times$ $10^{-20}[3-5]$ and " $\ldots$ system sensitivity could be increased by several orders of magnitude." However, there is no further planned development of the INFN Genoa HFRGW detector.

The third detector is the Kawamura $100 \mathrm{MHz}$ HFRGW detector that has been built by the Astronomical Observatory of Japan. It comprises two synchronous interferometers having arms lengths of $75 \mathrm{~cm}$. Miniaturization of the detector to $10 \mathrm{GHz}$ wavelengths would be $100: 1$.Its sensitivity is $h \approx 10^{-20}$, projected to improve to $\sim 10^{-23}$ for a $1000 \mathrm{~W}$ laser [6]. It appears that due to the small size of a miniaturized instrument and the lack of enough photons in the sampling period to perform accurate interferometry, it will be difficult for this design to operate satisfactorily at $10 \mathrm{GHz}$ with their projected sensitivity.

Another HFGW detector, under development at the Steinberg Astronomical Institute in Russia [12,13] detects gravitational waves by their action on an electromagnetic wave in a closed waveguide or resonator.

\subsection{New Measurement Technique}

An objective of this paper is to present the rationale behind a proposed and planned HFRGW detector utilizing a new measurement technique termed the "Li effect." This theory was first published in 1992 [8]. Subsequently, the $\mathrm{Li}$ effect has been developed further in nine later peer-reviewed research papers [9,32-39] and is scrutinized by Valentine Rudenko and Nikolai Kolosnitsyn of the Sternberg Astronomical Institute of Moscow State University [40]. The key results are summarized in ref. [9] and a detailed discussion of the detection mechanism is given in ref. [39] and presented in compact form in the Appendix. Often the Li effect is identified as a three- dimensional synchro-resonance electromagnetic coupling effect or 3D SR.

As mentioned in the Introduction this new detection technique is based upon coupling between an HFGW, a Gaussian-type microwave photon beam (having the same frequency, direction and suitable phase as the HFRGW being detected), and a static magnetic field. The result of this coupling is a flux of detection photons or perturbative photon flux (PPF), and reflectors would typically be used to direct the PPF towards sensitive microwave receivers [41]. First estimates of the Li-Baker detector's sensitivity in the microwave band have been similar to those needed for detection of primordial HFRGW [9,20, $27,39]$. There are, however, operational concerns such as fundamental noise sources that must be examined. Sources of noise in this detector include: background photon noise from the highly energetic Gaussian microwave beam (GB) including scattering, diffraction, thermal noise from the detector's containment vessel, dark-background shot noise, Johnson noise in the microwave receivers, preamplifier noise, and quantization noise.

In the Li-Baker-detector the key parameter is the first-order detection photons (proportional to GW strain amplitude $A$ ), or perturbative photon flux (PPF), and not the second-order PPF (proportional to $A^{2}$ ). The first-order $\mathrm{PPF}$, or the flux of detection photons produced by the Li-effect interaction with the GWs, is therefore proportional to $\sqrt{ } \Omega_{g w}$ and not $\Omega_{\mathrm{gw}}$. The spectra predicted by the pre-big-bang models (Figure 2 of [10]) shows that $\Omega_{g w}$ of relic GWs is almost constant at $6.9 \times 10^{-6}$ in the frequency range $=10 \mathrm{~Hz}$ to $10 \mathrm{GHz}$. Cosmic string models predict $\Omega_{g w} \sim 10^{-8}$ in the range $1 \mathrm{~Hz}$ to $10 \mathrm{GHz}$; its peak value is at about $10^{-7}$ to $10^{-6} \mathrm{~Hz}$, in the low-frequency regions - much lower than $\mathrm{HFGW}$ frequencies. Also, it is shown [10] that only the Advanced LIGO may achieve the requisite sensitivity for relic GWs predicted by the pre-big-bang model in the frequency band around $\sim 100$ $\mathrm{Hz}$; the present LIGO cannot detect relic GWs in that region. However, the Li-Baker detector could make observations of $h$ at around $10 \mathrm{GHz}$ and, unlike the current Low-Frequency Relic Gravitational Wave (LFRGW) detectors, could be sensitive enough to measure relic gravitational waves. Furthermore, with the dimensionless cosmological Hubble parameter $n=1.0$ and 1.2, there are sharp peaks of $\Omega_{g w}$ at $10 \mathrm{GHz}$ [42] as shown in Figure 1. Grishchuk's analyses that define these peaks are too lengthy to be included here, but can be found in Refs. [19,26-29]. A frequency scan, discussed in Section 4.5, could reveal other HFRGW effects of interest in the early universe at a variety of HFRGW base frequencies other than $10 \mathrm{GHz}$. 


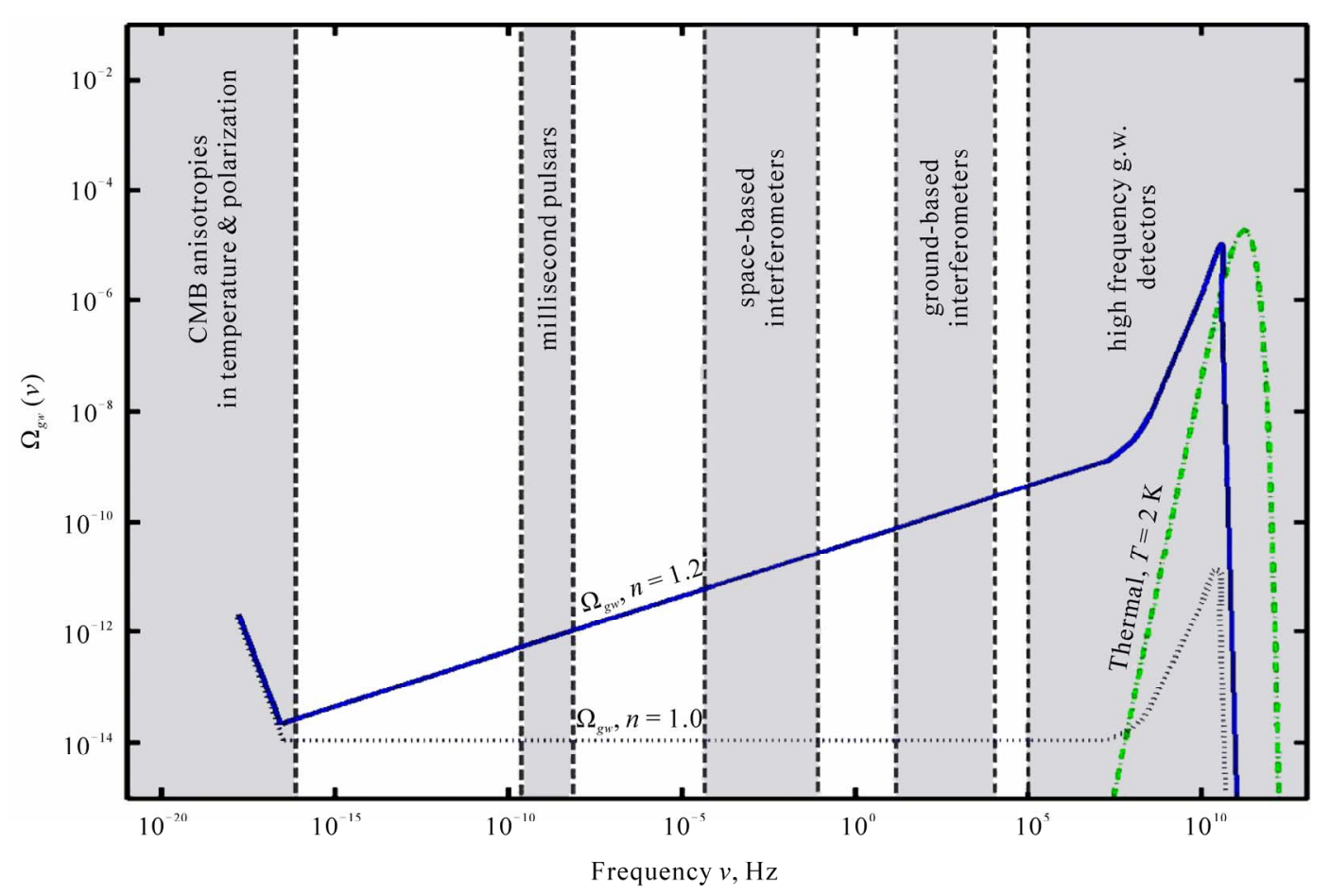

Figure 1. Predicted relic gravitational wave energy density as a function of frequency (slide 6, [42]).

\section{Electromagnetic and Gravitational Wave Interaction: The Gertsenshtein and $\mathrm{Li}$ Effects}

The Li Effect is very different from the well-known classical (inverse) Gertsenshtein effect [43], in which a GW travelling in a region in which there is a uniform constant applied magnetic field will produce a coupled electromagnetic (EM) wave having exactly the same frequency and wave-vector as the incoming GW. The coupled EM wave will exhibit a flux proportional to $A^{2}$. By contrast, in the Li effect, an electromagnetic (EM) traveling wave of a Gaussian beam (GB) in the presence of a perpendicular static magnetic field is found to interact with an incoming GW having exactly the same frequency and wave-vector (including the direction of propagation) as those of the EM wave and exhibit a flux proportional to $A$. This is known as the "synchro-resonance condition," which may typically be satisfied by one Fourier component of a continuous spectrum of incoming GWs. This interaction produces a resultant second EM wave of the same frequency as the EM and GW waves, but propagating perpendicular to both the applied uniform magnetic field and to the applied EM wave, as shown in Figure 2. It is unlike the (inverse) Gertsenshtein effect, in which the resultant EM wave is parallel to (rather than perpendicular to) the incoming GW, and in which there is no applied EM wave used to synchronized to the incoming GW.
There are two proven features of the Li effect (proof contained in the ten peer-reviewed references already cited [8,9,32-39], each covering a different aspect of the Li effect, and summarized in Appendix A). One is that the gravitational wave although transverse interacts with a Gaussian beam (GB) and the PPF do not travel in the same direction as the incoming gravitational wave. The second is that, regardless of direction, the PPF is a very low-impedance wave of order $A$ (e.g., for a HFGW of frequency $2.9 \mathrm{GHz}$ and amplitude $A \sim 10^{-30}$, the impedance of PPF is about $4.1 \times 10^{-11} \mathrm{ohms}$ ). By the way, the impedance of free space is $377 \mathrm{ohms}$ and the impedance of copper for an EM wave of frequency $30 \mathrm{GHz}$ is 0.02 ohms. In other words, free space looks like a very good "superconductor" to the PPF.

The perpendicular propagation direction of the PPF exhibited in Figure 2 is a fundamental physical requirement; otherwise the EM fields will not satisfy the Helmholtz equation, the electrodynamics equation in curved spacetime, the non-divergence condition in free space, and the laws of energy-conservation as discussed in the Appendix and in [35]. A significant feature of the Li-effect is that the PPF move both outward away from the GB's axis and inward toward the GB's axis. Thus reflectors in the GB itself could reflect and focus a portion of the PPF to microwave receivers in regions of the detector proper that are relatively noise free. The BPF noise, whatever its source (except for scattering as discussed in Section 5.1), mainly propagates radially out 


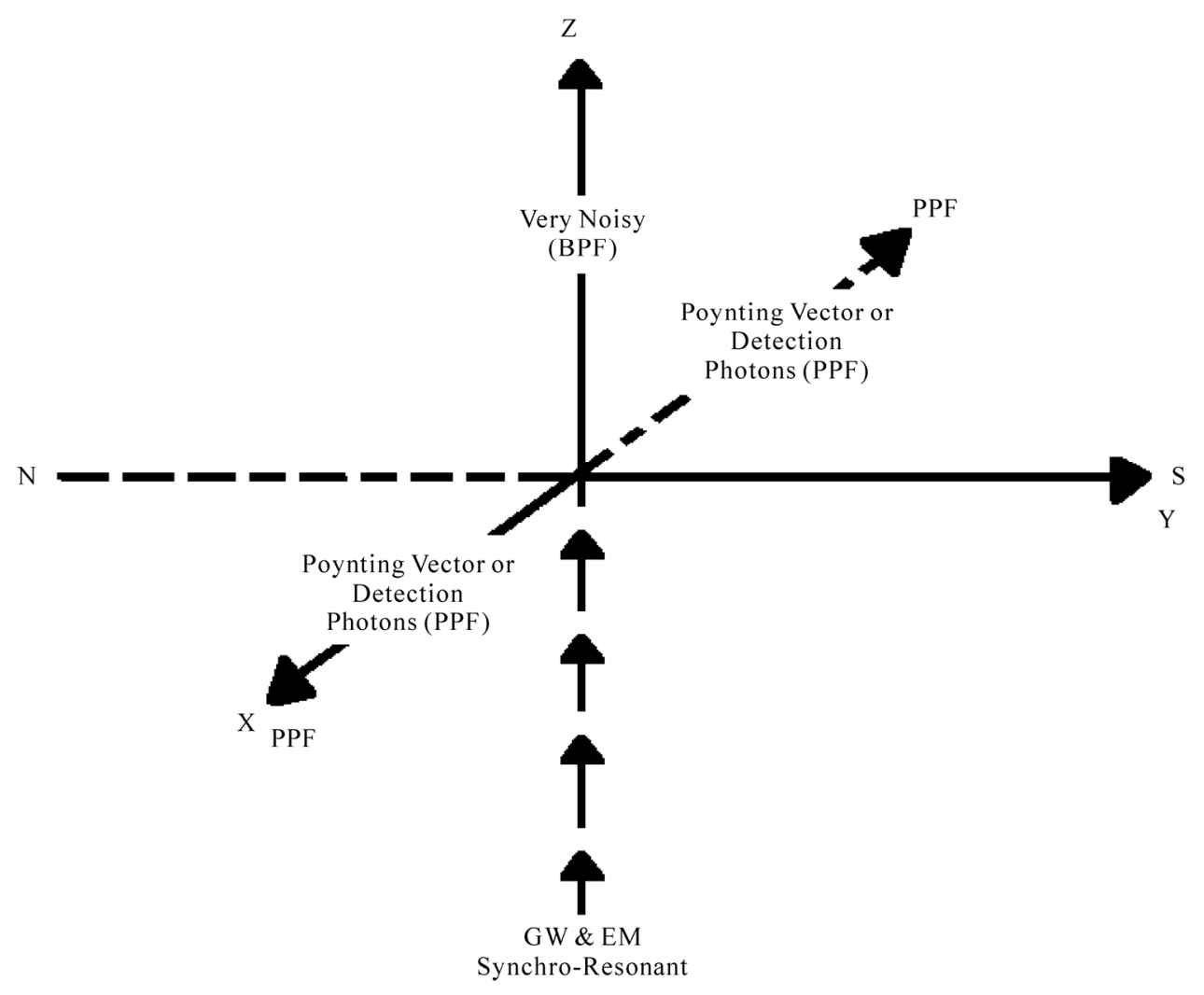

Figure 2. Li effect PPF-directed to the ends of the $x$-axis.

from the GB's axis and is not focused to the microwave receivers.

\section{Description of Li-Baker HFGW Detector and Its Physical Parameters}

\subsection{Gaussian Beam}

A traveling-wave Gaussian microwave beam (GB) is used as the applied EM wave required in the Li effect. In the Appendix is a more complete description of the GB. It is to be produced by a conventional microwave transmitter with its antenna aimed along the $+z$-axis of Figure 2. Its frequency and direction are the same as the frequency and direction of the incoming HFGW signal that is to be detected [44] as shown in Figure 3. The GB frequency is expected to be typically around $10 \mathrm{GHz}$ for GB directed along the $+z$-axis will allow detection of a HFRGW also directed along the $+z$-axis.

In order to reduce the thermal load on the refrigeration system the microwave transmitter and main GB microwave absorber are in separate chambers sealed off from the main detector chamber by microwave transparent walls. A high-vacuum system able to evacuate the chamber from $10^{-6}$ to $10^{-11}$ Torr (nominally about $7.5 \times$ $10^{-7}$ Torr) is needed to allow cryogenic operation and to reduce thermal noise (see Section 5.5).

\subsection{Magnetic Field and Sensitivity}

A static magnetic field $B$ (generated typically using one or more superconductor-magnets such as those found in a conventional MRI medical body scanner) is directed along the $y$-axis, as shown schematically in Figure 4. Rather than using one pair as shown schematically in Figure 4, it may be more cost-effective to use a number of magnet pairs spaced equally in the z-direction. The intersection of the magnetic field and the GB defines the "interaction volume" where the PPF is produced and move out in both $\mathrm{x}$ directions on both sides of the y-z-plane (as in Figure 2. A of the Appendix). The interaction volume in the GB for the proposed or nominal design is roughly cylindrical in shape, about $30 \mathrm{~cm}$ in length and about $9 \mathrm{~cm}$ cross-section diameter. In order to estimate the detection signal, or the number of detection photons (PPF) produced per second for a given amplitude HFGW, we will utilize Equation (7) of the analyses in [46], which is a simplification of Equation (59) in [34] for a near-field approximation as discussed in [46],

$$
n_{x}^{(1)}=\left[1 /\left(\mu_{0} \hbar \omega_{e}\right)\right] A B_{y} \psi_{0} \delta s
$$

where $n_{x}^{(1)}$ is the number of $x$-directed detection photons 


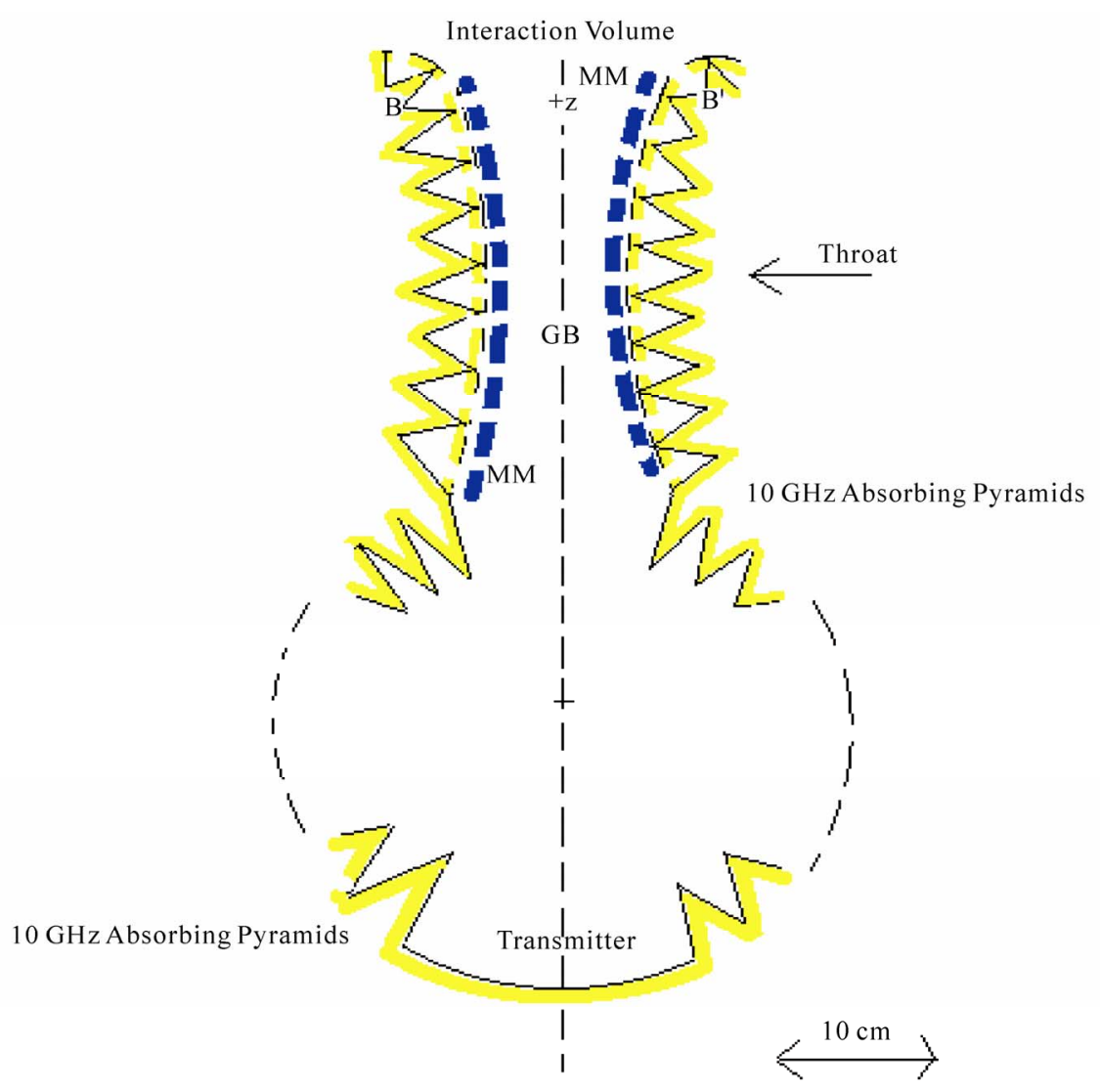

Figure 3. Gaussian-beam transmitter compartment.

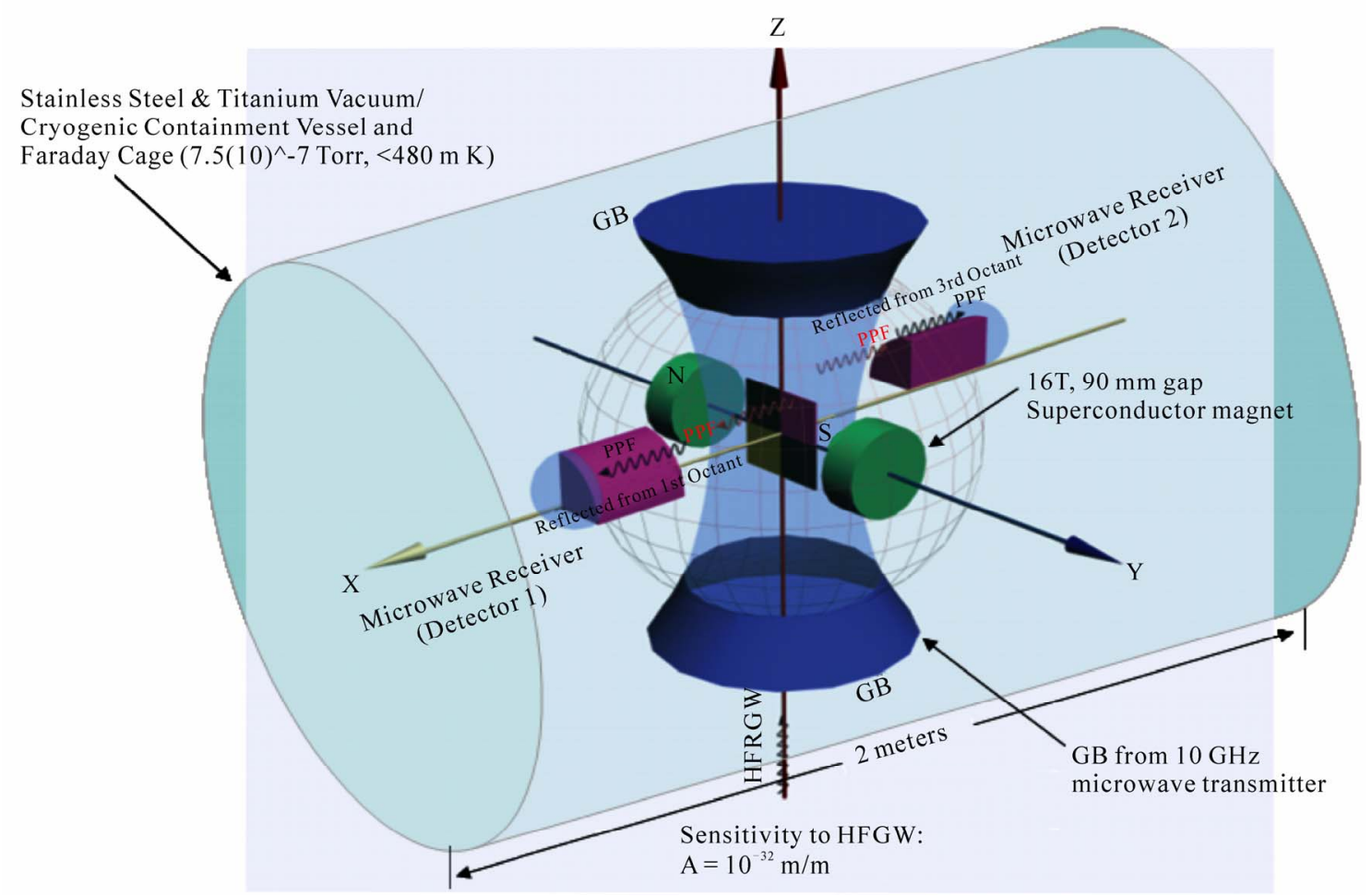

Figure 4. Schematic of the Li-Baker HFGW detector. 
per second produced in the interaction volume, $\hbar=$ Planck's reduced constant, $\omega_{e}=$ angular frequency of the EM wave $\left(=2 \pi v_{e}\right), v_{e}=$ frequency of the EM wave, $A=$ the HFGW amplitude, $B_{y}=y$-component of the magnetic field, $\psi_{0}=$ electrical field of the EM Gaussian beam and $\delta s$ is the cross-sectional area of the interaction volume perpendicular to the PPF. For the proposed nominal design, the minimum cross-section diameter or waist of the GB is located about $20 \mathrm{~cm}$ away from the antenna; the radius of the GB at its waist, $W$, is $\left(\lambda_{e} z / \pi\right)^{1 / 2}=4.4 \mathrm{~cm}$ at $10 \mathrm{GHz}$, so that its diameter is $8.8 \mathrm{~cm}$ (approximately the width of the interaction volume); and the length of the interaction volume is $l=30 \mathrm{~cm}$, so $\delta s=2 W l=2.58 \times$ $10^{-2} \mathrm{~m}^{2}$. From the analysis presented in ref. [32], the electrical field of the EM GB, $\psi$, is $1.26 \times 10^{4} \mathrm{Vm}^{-1}$ for transmitter power $1 \mathrm{~kW}$. For the present proposed design, $v_{e}=10^{10} \mathrm{~s}^{-1}, \omega_{e}=6.28 \times 10^{10} \mathrm{rad} / \mathrm{s}, A=10^{-30}$, and $B_{y}=$ $16 \mathrm{~T}$. Thus (1) gives $n_{x}^{(1)}=99.2 \mathrm{PPF}$ detection photons per second. For a $10^{3}$ second observation accumulation time interval, there would be about $10^{5}$ detection photons created (the PPF). About one-fourth of them would be focused at each receiver, since half would be directed towards $+x$ and half directed towards $-x$ on each side of the focusing reflectors in the $y-z$ plane (only the half of the photons directed toward the reflectors is focused to the microwave receivers the other half is directed away from the reflectors and unfocused and does not reach the receivers). Table 1 provides values for an interaction volume cross section of $\delta s=0.1 \mathrm{~m} \times 0.05 \mathrm{~m}=0.005 \mathrm{~m}^{2}$ (a very small detector), Table 2 is for $\delta s=0.30 \mathrm{~m} \times$ $0.088 \mathrm{~m}=0.0258 \mathrm{~m}^{2}$ (the proposed or nominal design) and Table 3 is for $\delta s=6 \mathrm{~m} \times 0.5 \mathrm{~m}=1.5 \mathrm{~m}^{2}$ (a large detector design). Table 3 is valid under the assumption that the near-field approximation of (1) still holds and account is taken of the spreading property of the GB. If the interaction volume is very large in one direction, for example much greater than $1 \mathrm{~m}$, then the computation of the total PPF could be somewhat more accurately obtained by a numerical integration of Equation (59) of [34], specifically, the numerical integration of the coefficients in equations (60) of [34]. In such a case the evacuation pressure would also need to be somewhat lower in order to increase the GB photon mean free path and minimize GB photon scattering (see Section. 5.1). Such a refinement is not judged to be necessary so the approximation of Equation (1) was utilized in Table 3. The proposed design or nominal case selected was a subjective judgment of the coauthors based upon their collective knowledge and experience with the cost, complexity of the fabrication of novel laboratory equipment and the availability of superconductor magnets and microwave transmitters and receivers.

It is again to be emphasized that unlike the Gertsen- shtein effect, the Li effect produces a first-order PPF whose amplitude is proportional to the incoming gravitational wave (GW) amplitude $A$ as in Equation (1) (and is not a second-order effect proportional to $A^{2}$ ). In the inverse Gertsenshtein effect, the EM wave produced is a second-order effect; from Equation (7) in [46], the number of EM photons produced in the inverse Gertsenshtein effect is "... proportional to the amplitude squared of the relic HFGWs, $A^{2}$," and it would be necessary to accumulate such EM photons for at least $1.4 \times 10^{16}$ seconds or 444 million years in order to achieve HFRGW detection utilizing the inverse Gertsenshtein effect as computed in [34]. Since in the Li effect the number of EM photons is proportional to the amplitude of the relic HFGWs, which is typically $A \approx 10^{-30}$, not its square, so that it would be necessary to accumulate such EM photons for at most about $10^{2}$ to $10^{5}$ seconds in order to achieve relic HFGW detection as computed in [34]. The JASON report [45] confuses the two effects and erroneously suggests that the Li-Baker HFGW detector utilizes the inverse Gertsenshtein effect. The Li-Baker HFGW detector does not utilize the inverse Gertsenshtein effect, and it has a theoretical sensitivity that is about $A / A^{2}=$ $10^{30}$ greater than the value incorrectly reported in the JASON report [45] for HFRGWs.

Table 1. PPF (photons per second) for various values of $B_{y}$ and transmitter power for $\delta s=0.005 \mathrm{~m}^{2}$.

\begin{tabular}{cccc}
\hline & Power $=100 \mathrm{~W}$ & Power $=1000 \mathrm{~W}$ & Power $=10,000 \mathrm{~W}$ \\
\hline$B_{y}=9 \mathrm{~T}$ & 3.4 & 10.8 & 34.2 \\
$B_{y}=16 \mathrm{~T}$ & 6.1 & 19.2 & 60.8 \\
$B_{y}=20 \mathrm{~T}$ & 7.6 & 24 & 76 \\
\hline
\end{tabular}

Table 2. PPF (photons per second) for various values of $B_{y}$ and transmitter power for $\delta s=0.0258 \mathrm{~m}^{2}$. The design or nominal case.

\begin{tabular}{cccc}
\hline & Power $=100 \mathrm{~W}$ & Power $=1000 \mathrm{~W}$ & Power $=10,000 \mathrm{~W}$ \\
\hline$B_{y}=9 \mathrm{~T}$ & 17.6 & 55.8 & 176.4 \\
$B_{y}=16 \mathrm{~T}$ & 31.4 & 99.2 & 313.7 \\
$B_{y}=20 \mathrm{~T}$ & 39.2 & 124 & 392
\end{tabular}

Table 3. PPF (photons per second) for various values of $\boldsymbol{B}_{\boldsymbol{y}}$ and transmitter power for $\delta s=1.5 \mathrm{~m}^{2}$.

\begin{tabular}{cccc}
\hline & Power $=100 \mathrm{~W}$ & Power $=1000 \mathrm{~W}$ & Power $=10,000 \mathrm{~W}$ \\
\hline$B_{y}=9 \mathrm{~T}$ & $1.023 \times 10^{3}$ & $3.2 \times 10^{3}$ & $1.026 \times 10^{4}$ \\
$B_{y}=16 \mathrm{~T}$ & $1.83 \times 10^{3}$ & $5.8 \times 10^{3}$ & $1.82 \times 10^{4}$ \\
$B_{y}=20 \mathrm{~T}$ & $2.3 \times 10^{3}$ & $7.2 \times 10^{3}$ & $2.3 \times 10^{4}$ \\
\hline
\end{tabular}


For an advanced Li-Baker detector [37], also included would be a resonance chamber $\left(Q \sim 10^{3}\right)$ in the interaction volume and more sensitive microwave receivers so that the sensitivity could be further improved. These refinements will be considered elsewhere.

\subsection{Microwave Reflectors}

Semi-paraboloid reflectors are situated back-to-back in the $y-z$ plane of the GB, as shown in Figures 5 and 6, to reflect the $+x$ and $-x$ propagating PPF to the microwave receivers. The effective aperture of each reflector is 60 $\mathrm{cm}$ and the sagitta or depth of curvature of such a mirror is about $2.26 \mathrm{~cm}$. Since this is greater than one tenth of a wavelength of the PPF, $\lambda_{e} / 10=0.3 \mathrm{~cm}$, such a paraboloid reflector is desirable rather than only a tilted plane mirror. As discussed in Section. 5, for elimination of any diffracted photons emanating from the GB's entrance to the main detector chamber at $B-B^{\prime}$ of Figure 5, the reflector's focus is below the $\mathrm{x}$ axis and "out of sight" of the GB's entrance. Thus the diffracted photons waves from the GB entrance will have at least one reflection from the absorbent detector walls prior to reaching the microwave receivers. As will be calculated in Section. 5 other radiation from the GB due to scattering and the natural fall off of $\mathrm{GB}$ radiation in the radial direction is negligible, so that the BPF is only due to diffraction from the transmitter's antenna, aperture or entrance to the main detector chamber. This is why the paraboloid mirrors are slightly tilted, which allows the focus to be slightly below the $x-y$ plane (similar to a Herschelian optical telescope) so that there is no direct straight line between the microwave receivers and the transmitting antenna. Since such a reflector would extend out $2.26 \mathrm{~cm}$ into the $\mathrm{GB}$ (on both sides of $y-z$ plane or $4.5 \mathrm{~cm}$ in total), a half or semi-paraboloid mirror is used instead in order not to block the Gaussian beam significantly. In the nominal case the reflectors are about $30 \mathrm{~cm}$ high (along the $\mathrm{z}$-axis) and $9 \mathrm{~cm}$ wide (along the $\mathrm{y}$-axis) and extend from $\mathrm{z}=0$ $\mathrm{cm}$ to $\mathrm{z}=+30 \mathrm{~cm}$ as shown in the figures. The reflectors can be installed inside the GB in order that the diffracted BPF from the GB transmitter's entrance to the detector chamber at $B-B^{\prime}$ and any diffraction perpendicular to the GB will not be directly focused onto the receivers. The only photons reflected or focused onto the microwave receivers will be the $\pm \mathrm{x}$-directed PPF photons in the GB that are directed toward the GB's center (there could be several microwave receivers stacked at each end of the $\mathrm{x}$-axis to in increase the field of view and account for any variations in the magnetic field from uniform straight lines). The semi-paraboloid reflectors are tilted "down" at about 1/2 $(3 \mathrm{~cm} / 100 \mathrm{~cm})=0.015$ radians (about $0.86^{\circ}$ ) or more (in order to focus at receivers $100 \mathrm{~cm}$ distant and $3 \mathrm{~cm}$ below the base of the GB) and extend from a sharp edge at point $A$ at the center of the GB, which is totally shielded from the re-

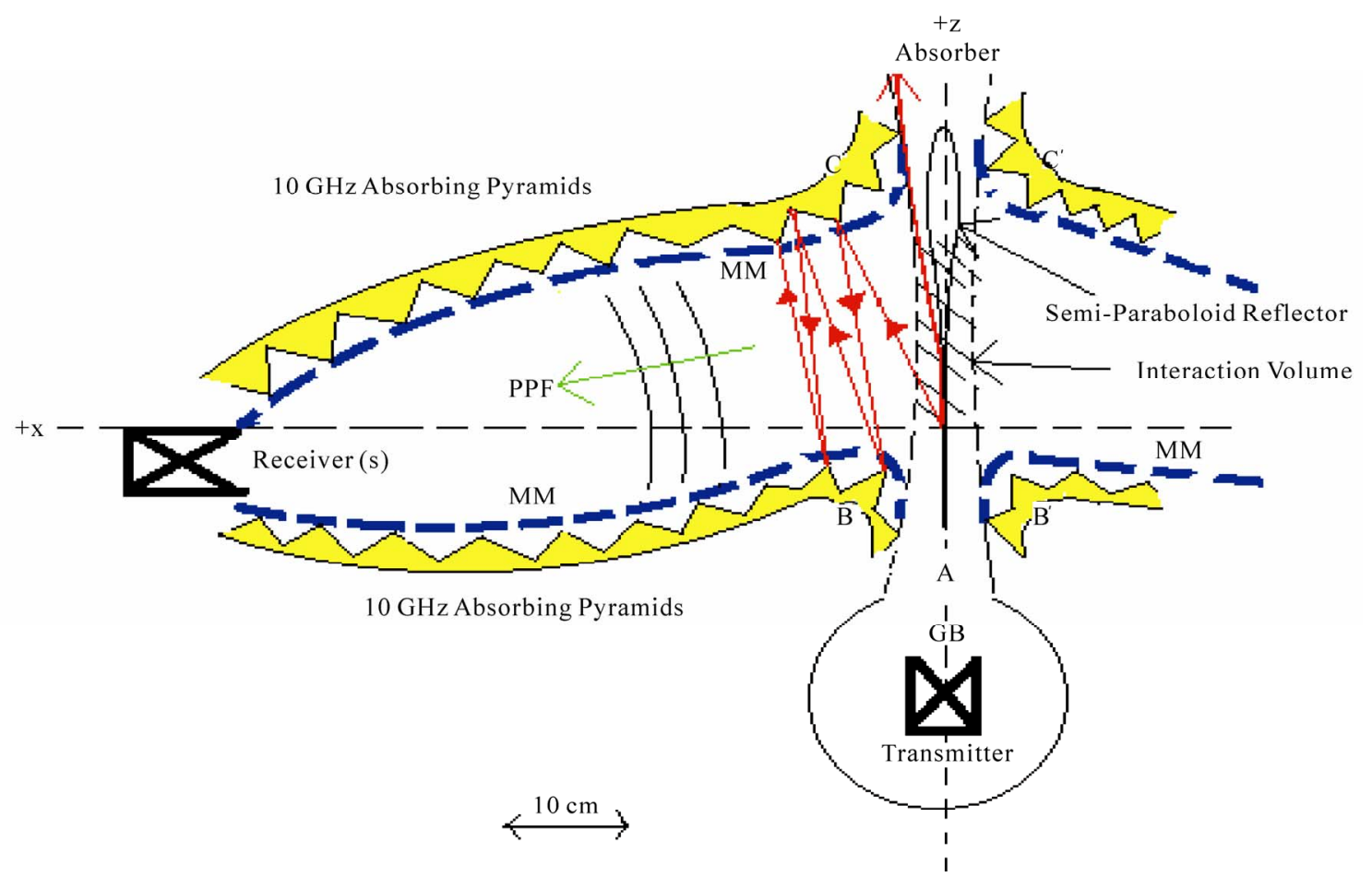

Figure 5. Side-view schematic of the Li-Baker HFGW detector, showing microwave-absorbent walls in the anechoic chamber and, if not totally absorbed, also showing the paths of reflected, diffracted photons. 


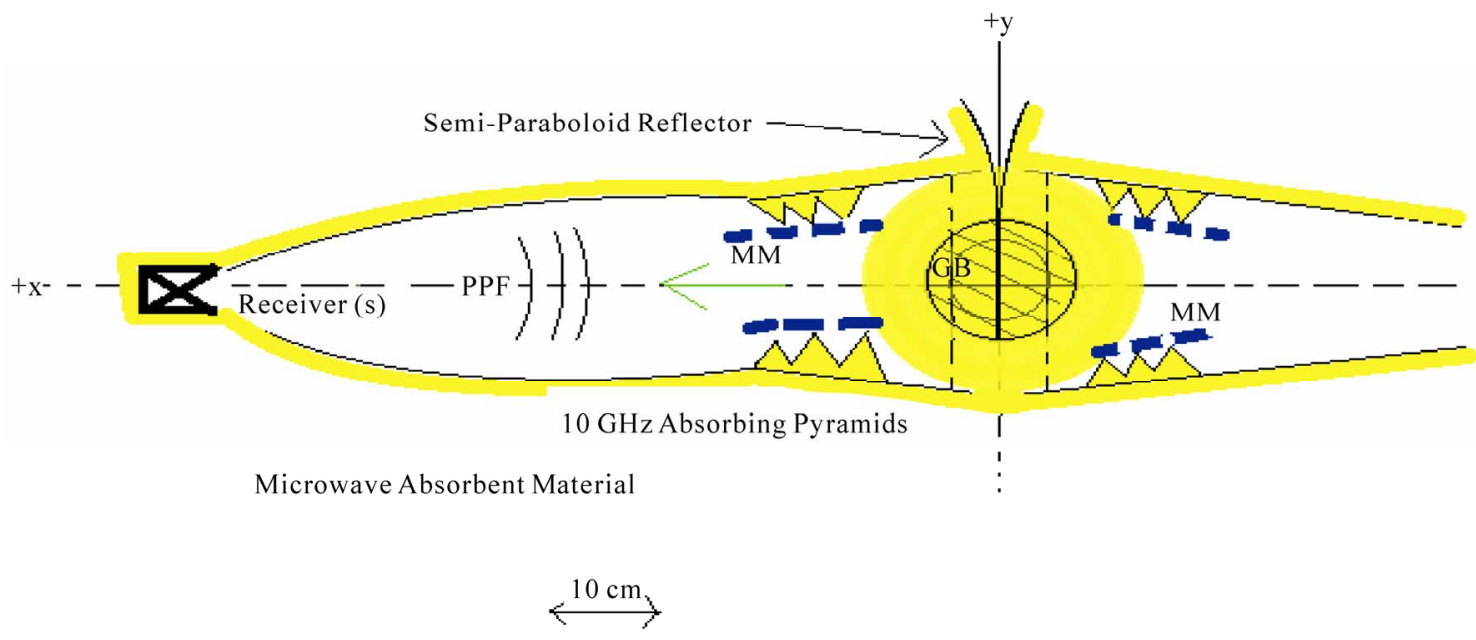

Figure 6. Plan-view schematic of the Li-Baker HFGW detector, exhibiting microwave-absorbent walls in the containment vessel and the reflectors extending out on either side of the $x$-axis along $y$ with edges completely shielded from the receivers.

ceivers, as shown in Figure 5. Thus there will be very little blockage of the GB. The reflectors can be constructed of almost any material that is non-magnetic (to avoid being affected by the intense magnetic field), reflects microwaves well and will not outgas in a high vacuum. The material of the reflectors can be in the form of fractal membranes that reflect more than $99 \%$ of the incident microwaves (experimental data from figure 1c of [47]). Apparently the fractal membranes (which consist of printed microcircuits) produce little diffraction in the presence of the GB and in the base frequency range pass all the remainder radiation through the fractal membranes [48]. Alternatively, microwave focusing lenses can be placed outside of the GB on either side [41] as shown in Figure 7.

\subsection{Microwave Receivers and Directionality}

High-sensitivity; shielded microwave receivers are located at each reflector focal point. Possible receiver technologies to use include a microwave horn plus receiver; a Rydberg Atom Cavity Detector [49]; a quantum electronics device (QED) microwave receiver, such as the Yale detector invented by Schoelkopf and Girvin [50], and a single-photon detector [51]. Such singlephoton microwave receivers or detectors can be refrigerated sufficiently to be unaffected by thermal-photon background or self noise. Of these receivers the microwave horn plus receiver is most likely for initial trials because of its off-the-shelf availability from many suppliers. The synchro-resonant condition specifies that the GW detected has the same frequency and propagation direction as the GB. In order to achieve a larger field of view and account for any curvature in the magnetic field, an array of microwave receivers having, for example, nine $3 \mathrm{~cm} \times 3 \mathrm{~cm}$ horns could be installed-parallel to the $y-z$ plane and $9 \mathrm{~cm}$ below the GB's base. Their field of view or directionality would be $9 \mathrm{~cm} / 100 \mathrm{~cm}=0.09$ radians or approximately five degrees.

\subsection{Bandwidth}

The "detected bandwidth" (BW) is determined by two fapctors:

- random fluctuations in the GB transmitter output causing BW broadening, and

- the bandwidth of the microwave receivers. In general, the narrower the frequency range or bandwidth is, the more sensitive is the detector (the noise floor is lowered at smaller BW).

However, frequency scanning allows a wide band of HFRGWs to be analyzed. As an example, in a $1 \mathrm{~Hz}$ "bandwidth" and a 1000 s observation interval, then over a year of observation about $30 \mathrm{kHz}$ HFRGW frequency band could be scanned. Essentially one would sample the detected BW by a number of very narrow actual bandwidths, $B_{w}$. If the observation interval is $1000 \mathrm{~s}$, then the actual $B_{w}$ is $0.001 \mathrm{~Hz}$. Or, for $100 \mathrm{~s}$ observation interval, then a $300 \mathrm{kHz}$ band of HFRGWs could be scanned. For a $1 \mathrm{kHz} \mathrm{BW}$, then a $0.3 \mathrm{GHz}$ band could be scanned using 100 s intervals over a year, and this would be a substantial BW if centered on $10 \mathrm{GHz}$ base frequency.

\section{Noise}

The Li-Baker HFGW detector contains a huge number of already existing EM quanta $\left(\sim 10^{26}\right.$ photons per second) in the intense GB. In addition to that there are noise sources in the detector that are similar to those encountered in any microwave receiver and may be analyzed in 


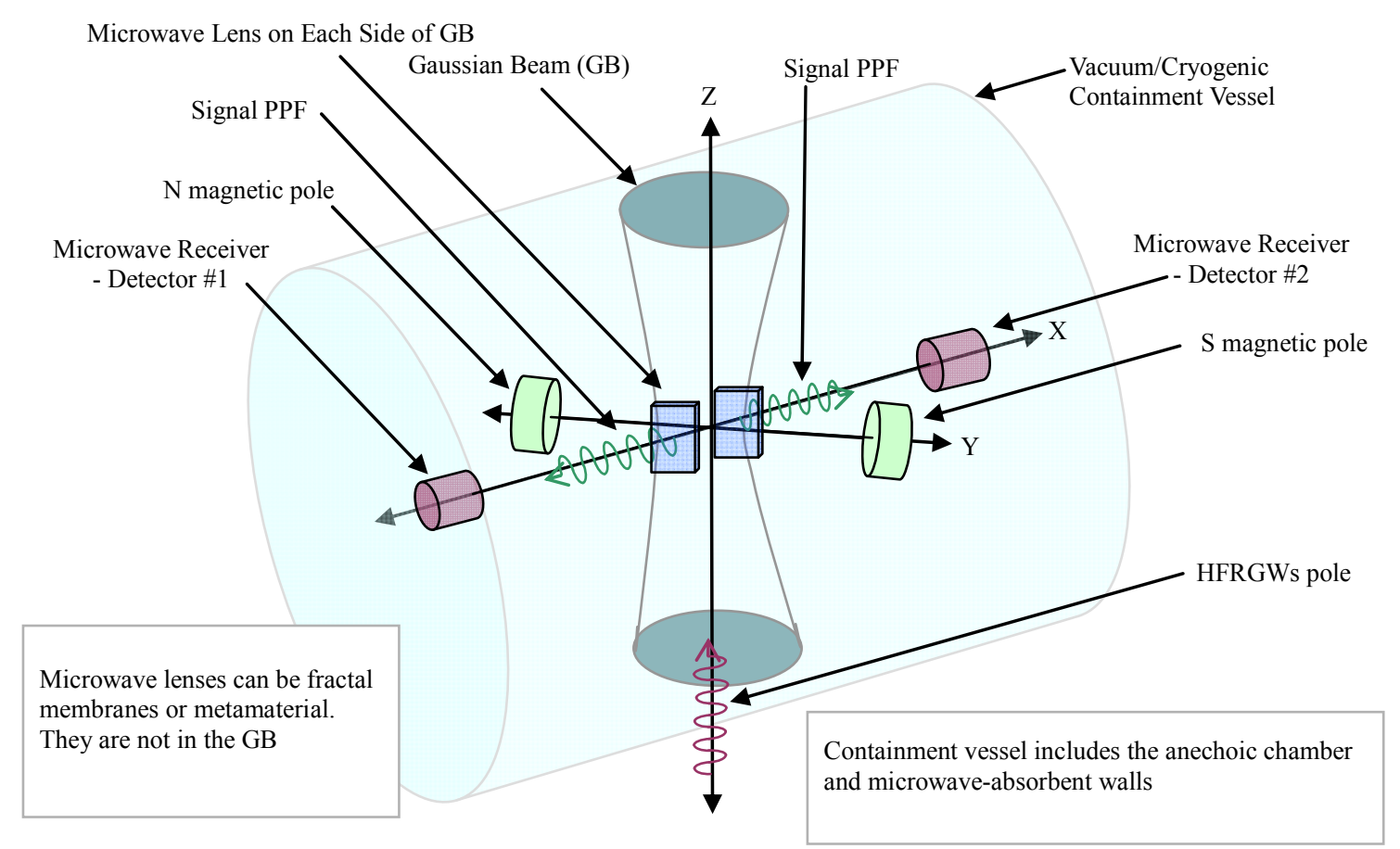

Figure 7. Schematic of the Li-Baker HFGW detector, exhibiting microwave lenses on each side of the GB focusing PPF on the microwave receivers.

similar fashion. The sensitivity of the detector depends critically on the noise at the microwave receivers. The background noise discussed in [35,39] did not consider the scattering or diffraction of the strong GB. However it will be shown that classical analyses of such scattering and diffraction [53-57] indicates that their effect will be negligible at the sensitive microwave receivers. In the Li-Baker detector the HFRGW signal manifests itself as detection photons (PPF) created by the interaction of a microwave beam (GB) and the GWs. The presence of the microwave beam having the same frequency as the detection photons gives rise to background photon flux (BPF) that produces dark-background shot noise such as scattering and diffraction, in addition to the usual microwave receiver noise. For example, Johnson noise originates thermally in any electrical resistor, and is often dominated by the contribution of the most significant resistance in the receiver input stage. In order to account for all these diverse noise sources, here they are translated through the detector to the actual microwave receiver(s) and usually termed noise equivalent power or NEP [52]. Photon noise from the GB will be considered in detail since it is likely to be the dominant source of noise in the Li-Baker detector.

\subsection{Noise Generated by the GB}

The intensity of the GB is written (Equation (3) of [36]) and is:

$$
n_{z}^{(0)} \sim \exp \left(-2 r^{2} / W^{2}\right)
$$

where $r$ is the radial distance out from the GB's axis and $W$ is the radius of the $\mathrm{GB}$ at its waist. The transverse $\mathrm{BPF}$ in any longitudinal symmetrical surface of the GB must vanish. Even if we treat a non-idealized situation, there are always the special local regions in which the transverse BPF vanish. If the transverse BPF in any longitudinal symmetrical surface of the GB is not vanishing, then the photon number at the symmetrical surface will be continuously accumulated (increased) with time in "the imploding wave" region of the GB and continuously reduced (decreased) with time in the "outgoing wave" region of the GB. Thus the "stability" of the GB would be destroyed (see Figures 2 and 4 on p. 414 of [9]). In the prototype Li-Baker HFRGW detector under analysis, which has peak sensitivity (base frequency) at $10 \mathrm{GHz}$, the energy per detection photon is $\hbar v_{e}=6.626 \times 10^{-24} \mathrm{~J}$, while the HFRGWs or the GB both have the same frequency for synchro-resonance. So a $10^{3} \mathrm{~W}$ GB flux is $1.51 \times 10^{26}$ photons/s. For 100-cm-distant microwave receivers, the GB intensity in the $z$-direction, if the classical Equation (2) is accurate at such large attenuations, is reduced to exp $\left(-2 \times 100^{2} / 4.4^{2}\right)\left(1.51 \times 10^{26}\right)$, which is essentially zero.

With regard to molecular scattering in the GB, we utilize the Rayleigh scattered intensity of microwave 
photons, $I$, from a molecule with incident photon intensity $I_{\mathrm{o}}$ as given by [53]

$$
I=I_{o} \frac{8 \pi^{4} \alpha^{2}}{\lambda^{4} R^{2}}\left(1+\cos ^{2} \theta\right)
$$

in which $\alpha$ is the atomic polarizability expressed as a polarization volume (where the induced electric dipole moment of the molecule is given by $4 \pi \varepsilon_{0} \alpha E$ ), $\theta$ is the scattering angle, and $R$ is the distance from particle to detector. Note that the scattering is not isotropic (there is a $\theta$-dependence), but in the present case, $\theta=90^{\circ}$ so the ratio of incident to scattered photon intensity is given by $\frac{8 \pi^{4} \alpha^{2}}{\lambda^{4} R^{2}}$. The polarizability is $\alpha \approx 1.1 \times 10^{-30} \mathrm{~m}^{3}$ from [54] so the scattering intensity ratio is $1.2 \times 10^{-49}$ for each atom in the chamber. The nominal volume of interaction is about $2000 \mathrm{~cm}^{3}$ (30 cm long and roughly $8 \mathrm{~cm} \times 8 \mathrm{~cm}$ in area) so at a pressure reduced to its convenient nominal value of $7.5 \times 10^{-7}$ Torr at temperature $480 \mathrm{mK}$, the number of molecules contained is about $3 \times 10^{16}$, giving a total scattering intensity ratio of $3.49 \times 10^{-33}$. There are $1.51 \times 10^{26}$ photons produced per second in the $10^{3} \mathrm{~W}$, $10 \mathrm{GHz}$ GB nominal case. Therefore, in $10^{3} \mathrm{~s}$ of observation time, the estimated number of photons received from Rayleigh scattering in the interaction volume is $\left(3.49 \times 10^{-33}\right)\left(1.51 \times 10^{26}\right)(1000)=5.3 \times 10^{-4}$ and will be negligible.

\subsection{Noise Generated by Diffraction}

Diffraction can potentially produce $x$-directed photons from a z-directed wave such as the GB in the absence of any GW interactions. This is potentially a problem for the Li-Baker detector design because the diffracted signal may either swamp the microwave receivers or else will represent a significant extraneous source of shot noise. Therefore, all sources of diffraction should be eliminated or at least minimized $[55,56]$. For example, the corners at $B$ and $B^{\prime}$ of Figure 5 should have radii of curvature in excess of two wavelengths $(6 \mathrm{~cm})$ and all small obstructions and corners should have radii greater than three wavelengths (e.g., $9 \mathrm{~cm}$ ) and the only edge of the focusing reflector at $\mathbf{A}$ will have its diffracted waves absorbed prior to reaching the receivers. In spite of this, there will be some microwave diffraction photon noise that will need to be reduced before reaching the receivers. Since there is no direct path perpendicular to the $G B$ to the microwave receivers in the $x$ direction or from the edges of the reflectors, due to the Herschelian optical telescope design, all $\mathrm{x}$-directed photons (moving perpendicular to the axis of the GB as computed in [57]) and all diffracted photons from the reflector edge will necessarily encounter a wall of the detection chamber before reaching the receivers.

The number of diffraction photons emanating radially from the GB, including the effect of polarization alignment, is given by Equation (13) of [57] where diffraction has been analyzed specifically for the Li-Baker detector. The reader is encouraged to review this important analysis since GB diffraction might be considered to be a major source of noise. The diffraction photons per second from the GB is

$$
n_{d i f}=k^{2}\left((d / 2)^{2} /\left(32 L_{d}^{2}\right)\right) \exp \left(-1 / 2 k^{2}[d / 2]^{2}\right)(0.01) n_{G B}
$$

where $k=2 \pi v_{e} / c$ (nominally, $209 \mathrm{rad} / \mathrm{m}$ at $10 \mathrm{GHz}$ ), $c$ being the speed of light, the diameter of the GB throat is $d(\sim 0.09 \mathrm{~m}$ for the nominal case, essentially $2 W), L_{d}$ is the distance of a receiver from the GB $(\sim 1 \mathrm{~m}$ for the nominal case) and $n_{G B}$ is the GB photon flux (nominally, $1.51 \times 10^{26}$ photons per second). The $n_{\text {dif }}$ decreases greatly with larger GB throat diameter. For example, for $d=0.06 \mathrm{~m}, n_{\text {dif }}=5.4 \times 10^{15}, d=0.09 \mathrm{~m} . n_{\text {dif }}=2.6 \times 10^{5}$, but for $d=0.12 \mathrm{~m}, n_{\text {dif }}=5.3 \times 10^{-10}$ diffraction photon per second. In this same regard please see Figure 4 of [57]. We will assume a single bounce or wave reflection of this diffraction-noise wave from the detector walls. The diffraction photon-path distance, prior to reaching the receivers, is $L_{d}(\sim 1 \mathrm{~m}$ for the nominal case). The number of diffraction photons, $n_{d i f}$, moving radially will be almost evenly spread out on an area of a band of a cylinder the width of which is the length of the GB, $l$ ( $\sim 0.3 \mathrm{~m}$ for the nominal case), having a spread of $\pi L_{d}$. Thus the number of diffracted noise photon impinging on each receiver per second, $n_{r d i f}$ is given by

$$
n_{\text {rdif }}=n_{\text {dif }}\left[a_{r} /\left(l \pi L_{d}\right)\right] \varepsilon_{a b}
$$

where $\varepsilon_{a b}$ is the wall absorption coefficient (e.g., for the nominal case to be discussed below, it would be $10^{-22}$ ) and $a_{r}=$ area of the square receiver horn or receiving surface (nominally, one HFRGW wavelength square or $\left.9 \times 10^{-4} \mathrm{~m}^{2}\right)$.

\subsection{Absorbing Walls}

The chamber wall absorbers are of two types: metamaterial or MM absorbers, which have no reflection, only transmission [58] at the base frequency and the usual commercially available absorbers in which there is reflection, but no transmission. In theory, multiple layers of metamaterials could result in a near "perfect" absorber (two MM layers absorbs noise to $99.9972 \%$ or $-45.5 \mathrm{~dB}$ over their specific base frequency range 5 to $10 \mathrm{GHz}$, according to the experimental data of Landy, et al. (page 3 of [58]). An absorbent "mat" combination of MMs 
(sketched as blue lines in Figures 3, 5 and 6) backed up by commercially available microwave absorbers is shown in Figure 8. As Landy, et al. [58] state in Physical Review Letters: "In this study, we are interested in achieving (absorption) in a single unit cell in the propagation direction. Thus, our MM structure was optimized to maximize the [absorbance] with the restriction of minimizing the thickness. If this constraint is relaxed, impedance matching is possible, and with multiple layers, a perfect [absorbance] can be achieved." We analyze an absorption mat (Patent Pending) consisting of two double MM layers, each double layer having a $-45 \mathrm{~dB}$ absorption. Behind the MM layers is a sheet of $10 \mathrm{GHz}$ tuned microwave pyramid absorbers, providing $-40 \mathrm{~dB}$ absorption (guaranteed) before reflection back into the MM layers. Thus the total absorption is $-45-45-40-45-45=$ $-220 \mathrm{~dB}$ or an absorption coefficient of $10^{-22}$ for the two double MM layers. There are several commercially available pyramid microwave absorbers available that offer the required low reflectivity, such as ARC Technologies, Cummings Microwave and the ETS Lindgren Rantec microwave absorbers. The ETS Lindgren EHP5PCL absorbing pyramids seem like a good choice. At normal incidence the typical reflectivity is down $-45 \mathrm{~dB}$ (guaranteed $-40 \mathrm{~dB}$ ). It is also important to note that the incident ray can have almost any inclination. As Service writes in his article published in SCIENCE [59] “... Sandia Laboratories in Albuquerque, New Mexico are developing a technique to produce metamaterials that work with [electromagnetic radiation] coming from virtually any direction." A substrate of conventional electromagnetic-radiation absorbing material, such as an array of pyramidal foam absorbers, may outgas into a high vacuum and reduce the capability of the vacuum-producing equipment to achieve a hard vacuum. The layers above the substrate will also serve to seal the absorbing and reflecting substrate from the external vacuum and, therefore, not reduce the capability of the detector's vacuum-producing equipment. The surface and voids of the pyramidal acoustical tiles substrate, upon which the MM layers are deposited, is formed from dielectric material to fill those voids as well as to fill the voids between the various MM layers. Thus the absorbing pyramids will be sealed from evaporation, sublimation or out gassing of the material composing them.

\subsection{Parametric Analysis}

In Tables 4-6 are to be found a parametric analysis of the diffraction photons per second (based upon classical diffraction equations) and noise equivalent power (NEP) for three alternative configurations of detector-wall absorbent mats, for GB aperture diameters, $d$, of 2, 3 (nominal)

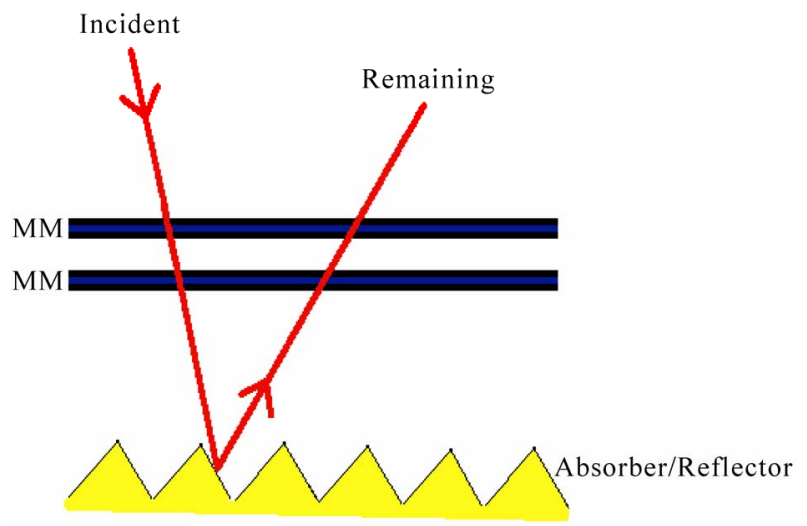

Figure 8. Schematic of typical multilayer metamaterial (two in the nominal case shown) or MM absorbers and pyramid absorber/reflector. Patent Pending.

Table 4. Diffraction photons $\mathrm{s}^{-1}$ and NEP $\mathrm{W}$ for a mat composed of absorbent microwave pyramids only, exhibiting an absorption of $-40 \mathrm{~dB}$.

\begin{tabular}{|c|c|c|c|}
\hline $\begin{array}{l}\text { GB aperture } \\
\text { diameter }\end{array}$ & $L_{d}=0.5 \mathrm{~m}$ & $\begin{array}{c}L_{d}=1.0 \mathrm{~m} \\
\text { (nominal) }\end{array}$ & $L_{d}=2.0 \mathrm{~m}$ \\
\hline$d=6 \mathrm{~cm}$ & $\begin{array}{l}3.4 \times 10^{6} \mathrm{~s}^{-1} \\
2.3 \times 10^{-17} \mathrm{~W}\end{array}$ & $\begin{array}{l}4.3 \times 10^{5} \mathrm{~s}^{-1} \\
3 \times 10^{-18} \mathrm{~W}\end{array}$ & $\begin{array}{l}5.3 \times 10^{4} \mathrm{~s}^{-1} \\
4 \times 10^{-19} \mathrm{~W}\end{array}$ \\
\hline $\begin{array}{l}d=9 \mathrm{~cm} \\
\text { (nominal) }\end{array}$ & $\begin{array}{l}3.3 \times 10^{-4} \mathrm{~s}^{-1} \\
2.2 \times 10^{-27} \mathrm{~W}\end{array}$ & $\begin{array}{l}4.2 \times 10^{-5} \mathrm{~s}^{-1} \\
2.8 \times 10^{-28} \mathrm{~W}\end{array}$ & $\begin{array}{l}5.2 \times 10^{-6} \mathrm{~s}^{-1} \\
3.5 \times 10^{-29} \mathrm{~W}\end{array}$ \\
\hline$d=12 \mathrm{~cm}$ & $\begin{array}{l}1 \times 10^{-18} \mathrm{~s}^{-1} \\
7 \times 10^{-42} \mathrm{~W}\end{array}$ & $\begin{array}{l}1.3 \times 10^{-19} \mathrm{~s}^{-1} \\
8.7 \times 10^{-43} \mathrm{~W}\end{array}$ & $\begin{array}{c}1.6 \times 10^{-20} \mathrm{~s}^{-1} \\
1 \times 10^{-43} \mathrm{~W}\end{array}$ \\
\hline
\end{tabular}

Table 5. Diffraction photons $\mathrm{s}^{-1}$ and NEP $\mathrm{W}$ for absorbent microwave pyramids and one MM layer (one layer of two $\mathrm{MMs})$, exhibiting an absorption of $-130 \mathrm{~dB}$.

\begin{tabular}{|c|c|c|c|}
\hline $\begin{array}{l}\text { GB aperture } \\
\text { diameter }\end{array}$ & $L_{d}=0.5 \mathrm{~m}$ & $\begin{array}{c}L_{d}=1.0 \mathrm{~m} \\
\text { (nominal) }\end{array}$ & $L_{d}=2.0 \mathrm{~m}$ \\
\hline$d=6 \mathrm{~cm}$ & $\begin{array}{l}3.4 \times 10^{-3} \mathrm{~s}^{-1} \\
2.3 \times 10^{-26} \mathrm{~W}\end{array}$ & $\begin{array}{c}4.3 \times 10^{-4} \mathrm{~s}^{-1} \\
3 \times 10^{-27} \mathrm{~W}\end{array}$ & $\begin{array}{c}5.3 \times 10^{-5} \mathrm{~s}^{-1} \\
4 \times 10^{-28} \mathrm{~W}\end{array}$ \\
\hline $\begin{array}{l}d=9 \mathrm{~cm} \\
\text { (nominal) }\end{array}$ & $\begin{array}{l}3.3 \times 10^{-13} \mathrm{~s}^{-1}, \\
2.2 \times 10^{-36} \mathrm{~W}\end{array}$ & $\begin{array}{l}4.2 \times 10^{-14} \mathrm{~s}^{-1} \\
2.8 \times 10^{-37} \mathrm{~W}\end{array}$ & $\begin{array}{l}5.2 \times 10^{-15} \mathrm{~s}^{-1} \\
3.5 \times 10^{-38} \mathrm{~W}\end{array}$ \\
\hline$d=12 \mathrm{~cm}$ & $\begin{array}{l}1 \times 10^{-27} \mathrm{~s}^{-1}, \\
7 \times 10^{-51} \mathrm{~W}\end{array}$ & $\begin{array}{l}1.3 \times 10^{-28} \mathrm{~s}^{-1} \\
8.7 \times 10^{-52} \mathrm{~W}\end{array}$ & $\begin{array}{c}1.6 \times 10^{-29} \mathrm{~s}^{-1} \\
1 \times 10^{-52} \mathrm{~W}\end{array}$ \\
\hline
\end{tabular}

Table 6. Diffraction photons $\mathrm{s}^{-1}$ and NEP $\mathrm{W}$ for absorbent microwave pyramids and four (two layers of two) MM layers (nominal), exhibiting an absorption of $-220 \mathrm{~dB}$.

\begin{tabular}{|c|c|c|c|}
\hline $\begin{array}{c}\text { GB aperture } \\
\text { diameter }\end{array}$ & $L_{d}=0.5 \mathrm{~m}$ & $\begin{array}{c}L_{d}=1.0 \mathrm{~m} \\
\text { (nominal) }\end{array}$ & $L_{d}=2.0 \mathrm{~m}$ \\
\hline$d=6 \mathrm{~cm}$ & $\begin{array}{l}3.4 \times 10^{-12} \mathrm{~s}^{-1} \\
2.3 \times 10^{-35} \mathrm{~W}\end{array}$ & $\begin{array}{c}4.3 \times 10^{-13} \mathrm{~s}^{-1} \\
3 \times 10^{-36} \mathrm{~W}\end{array}$ & $\begin{array}{c}5.3 \times 10^{-14} \mathrm{~s}^{-1} \\
4 \times 10^{-37} \mathrm{~W}\end{array}$ \\
\hline $\begin{array}{l}d=9 \mathrm{~cm} \\
\text { (nominal) }\end{array}$ & $\begin{array}{l}3.3 \times 10^{-22} \mathrm{~s}^{-1} \\
2.2 \times 10^{-45} \mathrm{~W}\end{array}$ & $\begin{array}{l}4.2 \times 10^{-23} \mathrm{~s}^{-1} \\
2.8 \times 10^{-46} \mathrm{~W}\end{array}$ & $\begin{array}{l}5.2 \times 10^{-24} \mathrm{~s}^{-1} \\
3.5 \times 10^{-47} \mathrm{~W}\end{array}$ \\
\hline$d=12 \mathrm{~cm}$ & $\begin{array}{l}1 \times 10^{-36} \mathrm{~s}^{-1} \\
7 \times 10^{-60} \mathrm{~W}\end{array}$ & $\begin{array}{l}1.3 \times 10^{-37} \mathrm{~s}^{-1} \\
8.7 \times 10^{-61} \mathrm{~W}\end{array}$ & $\begin{array}{c}1.6 \times 10^{-38} \mathrm{~s}^{-1} \\
1 \times 10^{-61} \mathrm{~W}\end{array}$ \\
\hline
\end{tabular}


and $4 \mathrm{~GB}$ microwave wavelengths $(6,9$, and $12 \mathrm{~cm})$ and for single-reflection diffraction path lengths, $L_{d}$, of 0.5 , 1.0 (nominal) and $2.0 \mathrm{~m}$ from the GB throat to the receivers. These distances are approximately the distance along the $\mathrm{x}$-axis of the microwave receivers from the axis of the GB. The nominal design choice is discussed in Section 4.2.

Note that if during prototype-detector tests it became apparent that diffraction rays reached a microwave receiver without being intercepted by an absorbent wall, then one would increase the diameter of the nominal or design GB from $9 \mathrm{~cm}$ to $12 \mathrm{~cm}$ resulting in diffraction flux at a receiver of $1.31 \times 10^{-15} \mathrm{~s}^{-1}$, which would be negligible. Such a design change would also increase detector size and cost, so this alternative design would not be pursued unless needed.

\subsection{Noise Generated by Thermal Photons}

In addition, isolation from background noise is further improved by cooling the detector chamber and proper choice microwave receiver apparatus [49-51] to reduce thermal noise background to a negligible amount. A cooling system is selected so that the temperature $T$ satisfies $k_{B} T \ll \hbar \omega$, where $k_{B}$ is Boltzmann's constant and $T \ll \hbar \omega / k_{B} \approx 480 \mathrm{mK}$ for detection at $10 \mathrm{GHz}$ and for the detector's narrow bandwidth. This condition is satisfied by the temperature for the detector enclosure $T<$ $480 \mathrm{mK}$, which can be conveniently obtained using a common helium-dilution refrigerator so that virtually no thermal photons from the chamber walls will be radiated at $10 \mathrm{GHz}$. According a study accomplished at the University of Western Australia "It is shown that this technology (new low noise microwave technology and ultra-cryogenic techniques) could measure the standard quantum limit of a macroscopic resonant-mass displacement detector." So that experimental data supports the reduction in thermal photons, through the use of modern refrigeration methods utilized in available microwave equipment [60], to allow high-sensitivity microwave detection.

\subsection{Comprehensive Noise Summary}

A standard sensor design method, already mentioned, for aggregating noise sources is to translate all noise terms through the system, or "refer them" from the location at which they occur to the equivalent noise or NEP detection photon microwave receiver (s) [52]. Such an expression of noise is equivalent to the amount of power that this amount of noise would represent at the detector. 11 the uncorrelated noise components can be root-sumsquared together, so that:

$$
\mathrm{NEP}=\sqrt{\left[\left(P_{n d}\right)^{2}+\left(P_{n s}\right)^{2}+\left(P_{n j}\right)^{2}+\left(P_{n p a}\right)^{2}+\left(P_{n q a}\right)^{2}\right]} \mathrm{W},
$$

where the equivalent-power noise components are defined as follows and the values for noise shown are based upon experimental data:

The dark-background shot noise is proportional to the square root of the number of photons present in a sample and is mitigated by using the absorption layers on the detector walls, larger GB throat diameter and wall geometry (Herschelian telescope geometry) to keep the microwave receivers "below" and "out of sight" of the GB entry-aperture source of diffraction and all $x$-directed diffraction from the GB kept "above" and not directed to the receivers as shown in Figure 5. (The x-directed diffraction from the GB move in planes parallel to the $x-y$ plane). Tables 4-6 present the calculated diffraction with the nominal design given in Table 5. Stray BPF spillover and diffraction that still manages to get reflected onto the detectors will create the shot noise, but such noise could be filtered out by pulse-modulating the magnetic field and a baffle arrangement shown in Figure 8.

The signal shot noise is $P_{n s}=\hbar v \sqrt{ }\left(N_{s}\right) / \Delta t$ where $N_{s}$ is the signal-photon count, and $\Delta t$ is the sample or accumulation time. This "noise" is part of the useful data and should not to be subject to elimination.

The Johnson noise (due to the thermal agitation of electrons when they are acting as charge carriers in a power amplifier) is $P_{n j}=4 k_{B} T R_{L} B_{W}$, [61] where $R_{L}$ is the equivalent resistance of the front-end amplifier and $B_{W}$ is the bandwidth. Mitigation of this noise source is accomplished by reducing bandwidth or reducing load resistance. However, in practice the bandwidth is often fixed by the application, in this case by the detection bandwidth. And the load resistance is required to generate a large voltage from a very small current. Hence there is in practice an optimum selection of load resistance that will optimize the signal to noise output during the initial tests of the Li-Baker detector, and the selection of this load resistance is the essence of impedance matching in its most basic form. Johnson noise is generally reduced or eliminated by refrigeration to $0.48 \mathrm{~K}$. At a $B_{w}$ of 0.001 $\mathrm{Hz}$ and a sample interval of $\Delta t=1000$ seconds the noise is $3.37 \times 10^{-28} \mathrm{~W}$ or $5 \times 10^{-5}$ noise photons per second [62].The preamplifier noise is $P_{n p a}=B_{w} / f_{1}$, [61] which is essentially $1 / f$ noise, where the crossover frequency $f$ is related to stray capacitance and load resistance; in which $f_{1}=1 /\left(2 \pi R_{L} C_{j n}\right),[61]$ where $C_{j n}=$ detection capacitance plus FET (field effect transistor) input capacitance plus stray capacitance. This noise source is mitigated by reducing bandwidth, reducing load resistance, or reducing stray capacitance. From [63] at a $B_{w}$ of $0.001 \mathrm{~Hz}$ and a sample interval of $\Delta t=1000$ seconds the noise is $7.57 \times$ 
$10^{-30} \mathrm{~W}$ or $1.13 \times 10^{-6}$ noise photons per second [64].

The quantization noise is $P_{n q a}=\mathrm{QSE} / \sqrt{12}$, where QSE is the quantization step equivalent or the value of one LSB (Least Significant Bit, the smallest value that is quantized by an ADC, or Analog to Digital Converter). This noise source is easily mitigated and eliminated by increasing the number of bits used in an ADC so that the LSB is a smaller portion of the overall signal. In practice the QSE is selected so that it does not cause lower SNR. The noise is $1.33 \times 10^{-26} \mathrm{~W}$ or $2 \times 10^{-3}$ noise photons per second.

The mechanical thermal noise is caused by the Brownian motion of sensor components. Mitigation is to refrigerate the sensing apparatus to reduce thermal inputs. The $0.48 \mathrm{~K}$ cooling should be sufficient, but if not an even lower temperature can be achieved [60,65]. Also, as mentioned earlier, there are specialty devices that could be made readily available internationally that meet the $0.48 \mathrm{~K}$ temperature considered for the nominal case [60].

The phase or frequency noise (of the EM-GB) is due to the fluctuations in the frequency of the microwave source for the GB. Steps will need to be taken during the Li-Baker detector tests to keep the GB source tuned precisely to the interaction volume resonance, thus reducing phase noise and maximizing the resonant magnification effect required from the interaction volume cavity. A cavity-lock loop or alternatively a phase-compensating feedback loop will be selected during post-fabrication trials to mitigate this noise source.

The noise or noise equivalent power at the receiver(s) or NEP as summarized in Table 7, is not a constant, but exhibits a stochastic or random component. In order to obtain the best estimate of the detection photons, one would need to utilize a conventional signal-processing filter [67].

The total NEP from Equation (5) of $1.02 \times 10^{-26} \mathrm{~W}$ (noise flux is $1.54 \times 10^{-3}$ photons per second) is Quantization and thermal noise limited at roughly $1 \times 10^{-26}$ to $2 \times 10^{-27} \mathrm{~W}$ for a detector temperature of $0.48 \mathrm{~K}$. If need be the receivers could be further cooled and shielded from noise by baffles [55] as shown in Figure 9 in which the spherical BPF wave front, if significant, can be reduced by baffle diffraction and the PPF focused by the reflectors passed through the baffle openings with less interaction with baffle edges and less diffraction. Given a signal that exhibits the nominal value given in Table 2 of $99.2 \mathrm{~s}^{-1}$ photons, one quarter of which is focused on each of the microwave receivers, which is $24.8 \mathrm{~s}^{-1}$ photons or $1.6 \times 10^{-22} \mathrm{~W}$, the signal-to-noise ratio for each receiver is better than $1500: 1$.

\subsection{Noise Mitigation by Magnetic-Field Modulation}

As noted, a unique feature of the Li-Baker HFGW detector is that some of the noise sources are present when the magnetic field is "off" and there is no signal or detection photons present. With the magnetic field "on" there is noise plus the signal. Thus, one can distinguish between HFRGW generated photons and the background generated photons from the GB. In principle one could use coincidence gating to subtract the "noise" (with the magnet "off") from the "signal plus noise" with the magnet "on" and obtain the signal alone. However, there will still be stochastic noise sources that form a noise spectrum that can be reduced by filtering but cannot be completely removed. Consider a simplified case of a uniform, low-frequency (compared with the $10 \mathrm{GHz}$

Table 7. Summary of Li-Baker detector noise based upon experimental data concerning its components.

\begin{tabular}{|c|c|c|c|}
\hline Noise Contributor & Brief Description of Noise source & Mitigation/Elimination Means & $\begin{array}{l}\text { Nominal Computed Value } \\
\text { photons } \mathrm{s}^{-1} \text {, NEP W }\end{array}$ \\
\hline Dark-background shot noise & GB noise especially diffraction & $\begin{array}{l}\text { Wall geometry and absorbing wall } \\
\text { materials }\end{array}$ & $4.2 \times 10^{-23} \mathrm{~s}^{-1}, 2.8 \times 10^{-46} \mathrm{~W}$ \\
\hline Signal shot noise & Noise in the signal itself & $\begin{array}{l}\text { Part of useful data and not to be } \\
\text { eliminated }\end{array}$ & -- \\
\hline Johnson noise & $\begin{array}{l}\text { Thermal agitation in a power } \\
\text { amplifier resistance }\end{array}$ & Refrigeration to low temperature & $5 \times 10^{-5} \mathrm{~s}^{-1}, 3 \times 10^{-28} \mathrm{~W}$ \\
\hline Preamplifier kTC noise & $\begin{array}{l}\text { Stray capacitance and load } \\
\text { resistance }\end{array}$ & $\begin{array}{l}\text { Reducing bandwidth, load } \\
\text { resistance and/or stray capacitance. }\end{array}$ & $1 \times 10^{-6} \mathrm{~s}^{-1}, 8 \times 10^{-30} \mathrm{~W}$ \\
\hline Quantization noise & Analog to Digital Converter & Increasing the number of bits used & $2 \times 10^{-3} \mathrm{~s}^{-1}, 1 \times 10^{-26} \mathrm{~W}$ \\
\hline Mechanical thermal noise & $\begin{array}{l}\text { Brownian motion of sensor } \\
\text { components. }\end{array}$ & Refrigeration to low temperature & $3 \times 10^{-4} \mathrm{~s}^{-1}, 2 \times 10^{-27} \mathrm{~W}$ \\
\hline Phase or Frequency noise & $\begin{array}{l}\text { Fluctuations in the frequency of the } \\
\text { microwave source for the GB. }\end{array}$ & $\begin{array}{l}\text { Cavity-lock loop or a } \\
\text { phase-compensating feedback loop }\end{array}$ & $5 \times 10^{-15} \mathrm{~s}^{-1}, 3 \times 10^{-38} \mathrm{~W}$ \\
\hline
\end{tabular}




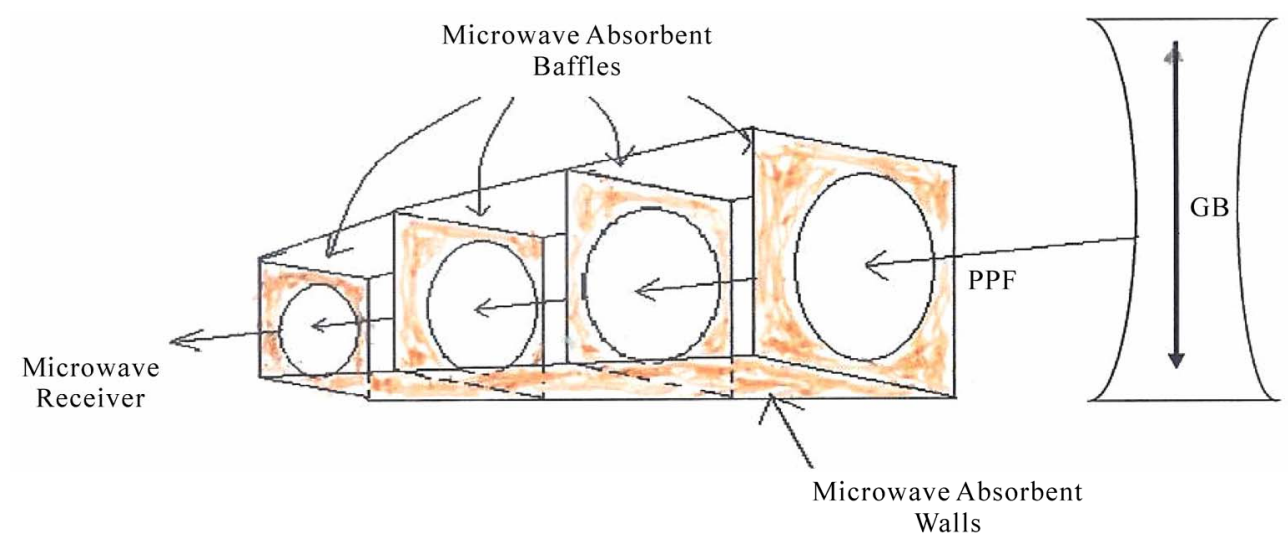

Figure 9. Schematic of microwave receiver shielded by MM absorbers and pyramid absorber/reflectors.

signal) square-wave chopper frequency energizing the magnet, with the magnet alternatively "off" and "on". It could be utilized to remove some of the background photons from the GB.

\subsection{Standard Quantum Limit (SQL)}

There is another possible concern here: Stephenson [68] concluded that a HFRGW intensity of $h_{\text {det }}=10^{-30}$ to $10^{-32} \mathrm{~m} / \mathrm{m}$ (time-varying strain in the fabric of space-time whose amplitude is $A$ ) represent the lowest possible GW strain variations detectable by each RF receiver in the Li-Baker HFGW detector. There is a limit to this sensitivity that is called "quantum back-action" or standard quantum limit (SQL) and is a result of the Heisenberg uncertainty principle [69]. An additional $(1 / \sqrt{ } 2)$ factor increase in maximum sensitivity applies if the separate outputs from the two RF receivers are averaged, rather than used independently for false alarm reduction, resulting in a minimum $h_{\text {det }}=1.2 \times 10^{-37}$. Because the predicted best sensitivity of the Li-Baker detector in its currently proposed configuration is $A=10^{-30} \mathrm{~m} / \mathrm{m}$, these results confirm that the Li-Baker detector is photon-signal-limited, not quantum-noise-limited; that is, the SQL is so low that a properly designed Li-Baker detector can have sufficient sensitivity to observe HFRGW of amplitude $A \approx 10^{-30} \mathrm{~m} / \mathrm{m}$ or less. In theory the Li-Baker detector is about seven orders of magnitude less sensitive than the standard quantum sensitivity limit.

\subsection{Sensitivity Increase}

It may be desirable to increase the sensitivity of the prototype Li-Baker detector through use of more sensitive microwave receivers, a stronger magnetic field, a more powerful microwave GB transmitter, a larger interaction volume, the introduction of resonance chambers, etc. In fact if the accumulation time, $\Delta t$, of the PPF at the re- ceivers is increased to $1000 \mathrm{~s}$, then for $A=10^{-33}$ the detection photons per second .would still be 99.2 from Equation (1). Of course the noise would also increase to 1.54 photons per second, but the signal to noise ratio would still exhibit a respectable value of 64. Even if one cannot greatly increase sensitivity immediately, a null experimental result at a larger value of $A$ would still be valuable, since it can provide the indirect means to determine whether or not some theories and scenarios should be corrected or eliminated. For example, the data analysis of low-frequency, laser-interferometer gravitational-wave detectors, such as LIGO and Virgo [70], have so far had null results, but have been the basis for cosmological theory improvements and have had important significance for further study.

\section{Conclusions}

Three HFGW detectors have previously been fabricated and two others theoretically proposed, but analyses of their sensitivity and the results provided herein suggest that for meaningful relic gravitational wave (HFRGW) detection, greater sensitivity than those fabricated instruments currently provide is necessary. The theoretical sensitivity of the Li-Baker HFGW detector studied herein, and based upon a different measurement technique than the other detectors, is predicted to be $A=$ $10^{-30} \mathrm{~m} / \mathrm{m}$ at base frequencies near to $10 \mathrm{GHz}$. This detector design is not quantum-limited and theoretically exhibits sensitivity sufficient for useful relic gravitational wave detection. Utilization of magnetic-field pulsed modulation allows for reduction in some types of noise. Other noise effects, based upon classical equations or experimental data, are found theoretically to be minimal; but they can only be accurately determined based on the Li-Baker prototype detector tests and some of the design and adjustments can only be finalized during prototype fabrication and testing. The detector can be built from 
off-the-shelf, readily available components and its research results would be complementary to the proposed low-frequency gravitational wave (LFGW) detectors, such as the Advanced LIGO and the proposed Laser Interferometer Space Antenna or LISA.

\section{Acknowledgements}

This work is supported by the National Nature Science Foundation of China under Grant No. 11075224, the Foundation of China Academy of Engineering Physics under Grant Nos. 2008T0401 and 2008T0402, Chongqing University Postgraduates Science and Innovation Fund No. 200811B1A0100299, GravWave ${ }^{\circledR}$ LLC, Transportation Sciences Corporation and Seculine Consulting. All authors reviewed, edited and approved the manuscript. Some material by RCW was based on work supported by the US National Science Foundation, while working at the Foundation. Any opinion, finding, and conclusions or recommendations expressed in this material are those of the authors and do not necessarily reflect the views of the US National Science Foundation. FYL initially developed the theory; RMLBjr designed the microwave-absorbing baffles and the reflectors including the off-axis Herschelian telescope and the exterior to GB focusing lenses to reduce diffraction noise, suggested the use of the vacuum and the utilization of two microwave detectors, AWB developed the astrophysical applications, RCW analyzed the magnet and Gaussian beam scattering, GVS developed the standard quantum limit and the noise equivalent power approach and RCW and GVS developed the concept for the high-sensitivity microwave receivers and signal processing. Christine S. Black provided the figures and Amara D. Angelica edited the manuscript.

\section{References}

[1] A. M. Cruise, "An Electromagnetic Detector for VeryHigh-Frequency Gravitational Waves," Classical and Quantum Gravity, Vol. 17, No. 13, 2000, pp. 2525- 2530. doi:10.1088/0264-9381/17/13/305

[2] A. M. Cruise, et al., "A Correlation Detector for Very High Frequency Gravitational Waves," Classical and Quantum Gravity, Vol. 22, 2005, pp. 5479-5481. doi:10.1088/0264-9381/22/10/046

[3] P. Bernard, et al., "A Detector of Small Harmonic Displacement Based on Two Coupled Microwave Cavities," Review of Scientific Instruments, Vol. 72, No. 5, 2001, pp. 2428-2437. doi:10.1063/1.1366636

[4] A. Chincarini, et al., "Micro-Wave Based High-Frequency Gravitational Wave Detector," Paper HFGW03-103 Gravitational-Wave Conference, The Mitre Corporation, 2003.
[5] R. Ballantini, et al., "Microwave Apparatus for Gravitational Waves Observation," Istituto Nazionale Di Fisica Nucleare Technical Note INFN/TC-05/05, 2005.

[6] A. Nishizawa, et al., "Laser-Interferometric detectors for Gravitational Wave Backgrounds at $100 \mathrm{MHz}$ : Detector Design and Sensitivity," Physical Review D, Vol. 77, No. 2, 2007, p. 022002. doi:10.1103/PhysRevD.77.022002

[7] R. M. L. Baker Jr., "Gravitational Wave Generator, (Detector Portion)," Peoples Republic of China Patent Number 0510055882.2, 2007.

http://www.drrobertbaker.com/docs/Chinese\%20Detector $\% 20$ Patent.pdf

[8] F. Y. Li, "Interaction between Narrow Wave Beam-Type High Frequency Gravitational Radiation and Electromagnetic Fields," Acta Physica Sinica, Vol. 41, No. 12, 1992, pp.1919-1928.

[9] F. Y. Li, et al., "Perturbative Photon Fluxes Generated by High-Frequency Gravitational Waves and Their Physical Effects," The European Physical Journal C, Vol. 56, No. 3, 2008, pp. 407-423.

doi:10.1140/epjc/s10052-008-0656-9

http://www.drrobertbaker.com/docs/Li-Baker\%20Chines e\%20HFGW\%20Detector.pdf

http://www.gravwave.com/docs/Li-Baker\%206-22-08.pdf

[10] B. P. Abbott, et al., "An Upper Limit on the Stochastic Gravitational-Wave Background of Cosmological Origin," The LIGO Scientific Collaboration \& the Virgo Collaboration, Nature, Vol. 460, No. 7258, 2009, p. 990994.

[11] M. Krauss, "Primordial Gravitational Waves and Cosmology," Science, Vol. 328, No. 5981, 2010, pp. 989992. doi:10.1126/science. 1179541

[12] M. B. Mensky, "Problems of the Theory of Gravity and Elementary Particles,” No. 6, In: K. P. Stanyukovich, Ed., Atomizdat, Moscow, 1975, pp. 181-190.

[13] M. B. Mensky, et al., "High-Frequency Gravitational Wave Detector with Electromagnetic-Gravitational Resonance," Gravitation and Cosmology, Vol. 15, No. 2, 2009, pp. 167-170. doi:10.1134/S0202289309020133

[14] A. Einstein, "Die Feldgleichungen der Gravitation," Sitzungsberichte der Preussischen Akademie der Wissenschaften zu Berlin, 1915, pp. 844-847. http://nausikaa2.mpiwg-berlin.mpg.de/cgi

[15] R. Brustein, et al., "Relic Gravitational Waves from String Cosmology," Physical Letters B, Vol. 361, No. 1-4, 1995, p. 45. doi:10.1016/0370-2693(95)01128-D

[16] A. Buonanno, et al., "Spectrum of Relic Gravitational Waves in String Cosmology," Physical Review D, Vol. 55, No. 6, 1997, Article ID 03330. doi:10.1103/PhysRevD.55.3330

[17] H. J. de Vega, et al., "Generation of Gravitational Waves by Generic Sources in de Sitter Space-Time," Physical Review D, Vol. 60, No. 4, 1999, Article ID 04407. doi:10.1103/PhysRevD.60.044007

[18] M. Giovannini, "Production and Detection of Relic Gravitons in Quintessential Inflationary Models," Physical Review D, Vol. 60, No. 12, 1999, Article ID 01235. 
[19] L. P. Grishchuk, "34th Rencontres de Moriond: Gravitational Waves and Experimental Gravity," 1999.

[20] A. W. Beckwith, "Proceedings of the Space, Propulsion and Energy Sciences International Forum (SPESIF)," In: G. Robertson, Ed., Vol. 1103, American Institute of Physics Conference Proceedings, Melville, New York, 2009, p. 571.

[21] M. P. Infante, et al., "The Primordial Gravitation Wave Background in String Cosmology," Physical Review D, Vol. 61, 2000, Article ID 083515. doi:10.1103/PhysRevD.61.083515

[22] H. J. Mosquera-Cuesta, et al., "Bursts of Gravitational Radiation from Superconducting Cosmic Strings and the Neutrino Mass Spectrum," Physical Letters B, Vol. 500, No. 3-4, 2001, pp. 215-221. doi:10.1016/S0370-2693(01)00073-9

[23] G. S. Bisnovatyi-Kogan, et al., "Very High Frequency Gravitational Wave Background in the Universe," Classical and Quantum Gravity, Vol. 21, No. 14, 2004, pp. 3347-3359. doi:10.1088/0264-9381/21/14/001

[24] P. S. Shawhan, "Gravitational Waves and the Effort to Detect Them," American Scientist, Vol. 92, No. 4, 2004, pp. 350-356.

[25] A. W. Beckwith, http://vixra.org/abs/1009.0020, 2010.

[26] L. P. Grishchuk, "Primordial Gravitons and the Possibility of Their Observation," Journal of Experimental and Theoretical Physics Letters, Vol. 23, 1976, p. 293.

[27] L. P. Grishchuk, "Gravitational Primordial Gravitons and the Possibility of Their Observation," Journal of Experimental and Theoretical Physics Letters, Vol. 23, 1977, p. 293.

[28] L. P. Grishchuk, "Gravitational-Wave Astronomy," Soviet Physics Uspekhi, Vol. 31, 1988, p. 940. doi:10.1070/PU1988v031n10ABEH005634

[29] L. P. Grishchuk, arXiv: gr-qc/0504018v4, 2006.

[30] G. Garcia-Cuadrado, "Proceedings of the Space, Propulsion and Energy Sciences International Forum (SPESIF)," American Institute of Physics Conference Proceedings, In: G. Robertson, Ed., Vol. 1103, American Institute of Physics Conference Proceedings, Melville, New York, 2009, pp. 553-563.

http://www.gravwave.com/docs/Toward\%20a\%20New\% 20Era\%20in\%20Gravitational\%20Wave\%20Research.pd $\mathrm{f}$

[31] D. H. Douglass, et al., "General Relativity: An Einstein Centenary Survey," In: S. W. Hawking and W. Israel, Eds., CUP, UK, 1979, pp. 90-137.

[32] F. Y. Li, et al., "Electrodynamical Response of a High Energy Photon Flux to a Gravitational Wave," Physical Review D, Vol. 62, No. 4, 2000, Article ID 44018-1. doi:10.1103/PhysRevD.62.044018

[33] F. Y. Li, et al., "Electromagnetic Detection of HighFrequency Gravitational Waves," International Journal of Modern Physics D, Vol. 11, No. 7, 2002, pp. 10491059. doi:10.1103/PhysRevD.62.044018

[34] F. Y. Li, et al., "Electromagnetic Response of a Gaussian
Beam to High-Frequency Relic Gravitational Waves in Quintessential Inflationary Models," Physical Review D, Vol. 67, 2003, Article ID 104008-1-17.

[35] F. Y. Li, et al., "Coupling of a Linearized Gravitational Wave to Electromagnetic Fields and Relevant Noise Issues," Chinese Physical Letters, Vol. 20, No. 11, 2003, p. 1917. doi:10.1088/0256-307X/20/11/004

[36] F. Y. Li, et al., "Resonant Interaction between a Weak Gravitational Wave and a Microwave Beam in the Double Polarized States through a Static Magnetic Field," Chinese Physical Letters, Vol. 21, No. 11, 2004, p. 2113. doi:10.1088/0256-307X/21/11/011

[37] F. Y. Li, et al., "Detection of High-Frequency Gravitational Waves by Superconductors," International Journal of Modern Physics B, Vol. 21, No. 18-19, 2007, pp. 32743278. doi:10.1142/S0217979207044366

[38] F. Y. Li, et al., "Phase and Polarization State of High-Frequency Relic Gravitational Waves," Chinese Physical Letters, Vol. 26, No. 5, 2009, p. 5-050402.

[39] F. Y. Li, et al., "Perturbative Photon Fluxes Generated by High-Frequency Gravitational Waves and Their Physical Effects," The European Physical Journal C, Vol. 56, No. 3, 2009, pp. 407-423.

doi:10.1140/epjc/s10052-008-0656-9

http://www.drrobertbaker.com/docs/Li-Baker\%20Chines e\%20HFGW\%20Detector.pdf http://www.gravwave.com/docs/Li,\%20et\%20al.\%20PR D\%2009-9-09\%20.pdf

[40] N. Kolosnitsyn, "Personal Communication," 15 August 2010.

[41] R. M. L. Baker Jr., et al., "Proposed Ultra-High Sensitivity HFGW Detector," In: M. S. El-Genk, Ed., Proceedings of Space Technology and Applications International Forum (STAIF-2008), Vol. 968, American Institute of Physics Conference Proceedings, Melville \& New York, 2008, pp. 1045-1054.

http://www.gravwave.com/docs/Proposed\%20Ultra High\%20Sensitivity\%20HFGW\%20Detector\%2005-15-0 8.pdf

[42] L. P. Grishchuk, Proceedings of 2nd High-Frequency Gravitational Wave Symposium, Austin, No. 6, 19 September 2007.

http://www.gravwave.com/docs/Grishchuk\%20\%20HFG W\%20Lect.pdf

[43] M. E. Gertsenshtein, "Wave Resonance of Light and Gravitational Waves Soviet," Physics JETP, Vol. 14, No. 1, 1962, pp. 84-85.

[44] A. Yariv, "Quantum Electronics," 2nd Edition, Wiley, New York, 1975.

[45] D. Eardley, D JSR-08-506, the JASON Defense Science Advisory Panel and Prepared for the Office of the Director of National Intelligence, 2008.

[46] R. M L Baker, Jr., et al., "Piezoelectric-Crystal-Resonator High-Frequency Gravitational Wave Generation and Synchro-Resonance Detection," In: M. S. El-Genk, Ed., Proceedings of Space Technology and Applications International Forum (STAIF-2008), Vol. 813, American Institute of Physics Conference Proceedings, Melville \& 
New York, 2008, pp. 1280-1289.

[47] W. Wen, et al., "Sub Wavelength Photonic Band Gaps from Planar Fractals," Physical Review Letters, Vol. 89, No. 22, 2002, Article ID 223901. doi:10.1103/PhysRevLett.89.223901

[48] L. Zhou, et al., Physical Review Letters, Vol. 82, 2003, p. 7.

[49] K. Yamamoto, et al., arXiv:hep-ph/0101200 v1, 2001.

[50] D. Schuster, et al., arXiv:cond-mat/0608693 v1, 2006.

[51] G. S. Buller, et al., "Single-Photon Generation and Detection," Measurement Science and Technology, Vol. 21, No. 1, 2010, pp. 1-28. doi:10.1088/0957-0233/21/1/012002

[52] R. W. Boyd, "Radiometry and the Detection of Optical Radiation,” John Wiley \& Sons, New York, 1983.

[53] R. Nave, July 9, 2009. http://hyperphysics.phy-astr.gsu.edu/hbase/amos/blusky.h tml\#c2

[54] W. B. Robb, "The Frequency Dependent Polarizability of Atomic Nitrogen," Journal of Physics B: Atomic, Molecular and Optical Physics, Vol. 7, No. 13, 1974, p. L369. doi:10.1088/0022-3700/7/13/001

[55] J. B. Keller, "Geometrical Theory of Diffraction," Journal of the Optical Society of America, Vol. 32, No. 2, 1962, pp. 116-133. doi:10.1364/JOSA.52.000116

[56] C. J. Sheppard, et al., "Diffraction by a Circular Aperture: A Generalization of Fresnel Diffraction Theory," Journal of the Optical Society of America, Vol. A/9, No. 2, 1992, pp. 274-281.

[57] R. C. Woods, "Estimate of Diffraction from Gaussian Beam in Li-Baker HFGW Detector," In: G. Robertson, Ed., Proceedings of the Space, Propulsion and Energy Sciences International Forum (SPESIF), Melville \& New York, 2011. http://www.gravwave.com/docs/Woods\%202010.pdf

[58] N. I. Landy, et al., "Perfect Metamaterial Absorber," Physical Review Letters, Vol. 100, No. 20, 2008, pp. 207402-1-4. doi:10.1103/PhysRevLett.100.207402

[59] R. F. Service, "Next Wave of Metamaterials Hopes to Fuel the Revolution," Science, Vol. 327, No. 5962, 2010, pp. 138-139. doi:10.1126/science.327.5962.138

[60] M. E. Tobar, "Applications of Low-Temperature Microwave Techniques to the Measurement of Gravity Waves and Quantum Measurement of Macroscopic Systems," Physics B: Condensed Matter, Vol. 280, No. 1-4, 2000, pp. 520-524. doi:10.1016/S0921-4526(99)01851-7
[61] R. W. Boyd, "Radiometry and the Detection of Optical Radiation," John Wiley \& Sons, New York, 1983.

[62] M. D. Tsai, et al., "A 5-GHz Low Phase Noise Differential Colpitts CMOS VCO," IEEE Microwave and Wireless Components Letters, Vol. 15, 2005, p. 327. doi:10.1109/LMWC.2005.847696

[63] F. Giannini, et al., "Research Contributions of the Alma m Mater Studiorum-University of Bologna," Proceedings of Generally Accepted Auditing Standards, Milano, September 2002, pp. 23-27.

http://amsacta.cib.unibo.it/149/

[64] M. D. Tsai, et al., "Colpitts CMOS VCO," IEEE Microwave And Wireless Components Letters, Vol. 15, 2005, p. 328.

[65] M. Planck, "The Theory of Heat Radiation," 2nd Edition, Blackiston Son \& Co., Philadelphia, 1914.

[66] A. O. Caldeira and A. J. Legget, "Physica a Statistical and Theoretical Physics," Vol. 121, No. 3, September 1983, pp. 587-616.

[67] R. M. L. Baker Jr., "Astrodynamics: Applications and Advanced Topics," Academic Press, New York, 1967, pp. 376-392.

[68] G. V. Stephenson, "Space, Propulsion and Energy Sciences International Forum," In: G. A. Robertson, Ed., Pro- ceedings of American Institute of Physics Conference, Vol. 1103, Springer-Verlag, New York, 2009, pp. 542- 547.

http://www.gravwave.com/docs/HFGW\%20Detector\%20 Sensitivity\%20Limit.pdf

[69] T. J. Kippenberg and K. J. Vahala, "Cavity Optomechanics: Back-Action at the Mesoscale," Science, Vol. 321, No. 5893, 2008, pp. 1172-1176. doi:10.1126/science. 1156032

[70] B. Abbott, et al., "Searching for a Stochastic Background of Gravitational Waves with the Laser Interferometer Gravitational Wave Observatory Astrophys," The Astrophysical Journal, Vol. 659. No. 2, 2007, pp. 918-930. doi: $10.1086 / 511329$

[71] P. F. Goldsmith, "Quasioptical Systems: Gaussian Beam Quasioptical Propagation and Applications," IEEE Press Series on RF and Microwave Technology, 1998.

[72] O. Mata-Mendez, "Diffraction and Beam-Diameter Measurement of Gaussian Beams at Optical and Micro- wave Frequencies," Optics Letters, Vol. 16, No. 21, 1991, pp. 1629-1631. doi:10.1086/511329

[73] J. A. Kong, "Electromagnetic Wave Theory," Cambridge University Press, Cambridge, 2000. 


\section{Appendix}

\section{Brief Summary of the Li-Effect Proof}

The Li-Baker detector is based upon the Li-effect and is a coupling system among a traveling-wave Gaussiantype microwave photon beam, a static magnetic field, HFGWs and fractal membranes (or other equivalent microwave optics to focus detection photons on microwave receivers) $[9,33,34,39]$. This synchro-resonance electromagnetic coupling scheme or 3D SR or Li effect is based upon a particular type of synchro-resonant microwave EM beam, which is a special beam exhibiting the direction, frequency and appropriate phase as the HFGW to be detected. That beam is a classical microwave Gaussian beam or GB in which the intensity falls off radially from the beam's axis according to a Gaussian curve, hence the name. The GB is a microwave beam having an hour-glass shape that is focused down to a narrow waist. We take the axis of the GB to be the $\mathrm{z}$-axis and the axis of the orthogonal static magnetic field to be the y-axis with the origin at the GB's waist. Below the origin $(z<0)$ the GB is converging and above the origin $(z>0)$ it is diverging. The characteristics of the GB, for example the orthogonal $\mathrm{E}$ and $\mathrm{B}$ vectors comprising the Poynting vectors of the EM-GB, are different in the $x-y$ plane cross sections of the GB. That is they are different in the four quadrants: $\mathrm{x}>0, \mathrm{y}>0 ; \mathrm{x}>0, \mathrm{y}<0 ; \mathrm{x}<0, \mathrm{y}>0$; $\mathrm{x}$ $<0, y<0$ of the GB. Several descriptions of a classical $\mathrm{GB}$ are available in the literature [44,70-73]. In the volume defined by the GB and magnetic field intersection there are eight octants to be considered: four for $z>0$ and four for $z<0$. The GB is different in each of these octants and the HFGWs passing through these octants interact differently with the combined GB and magnetic field in each of these octants. Note that we choose the GB of transverse polarized electric modes (which has non-vanishing magnetic component) and not the GB of transverse polarized magnetic models. These diverse interactions between HFGWs and the GB in the various octants are the essence of 3D SR or Li effect and the Li-Baker detector that makes use of the 3D SR or Li effect. What has been proven theoretically in the peer-reviewed literature [8,9,32-39] (each covering a different aspect of the Li-effect) is that the HFGWs passing through these octants generate microwave EM radiation or detection photons that move out in the $+x$ and $-x$ directions. In practice, due to the variety of directions of the actual HFGWs, they move out in a cone whose axis is the $\mathrm{x}$-axis and vertex is at the origin of coordinates. In order to concentrate or focus the microwave detection photons, whose presents signal the detection of HFGWs, at sensitive microwave receivers at both ends of the $\mathrm{x}$-axis, it is useful to introduce microwave optical devices. These devices can be in the form of concave metal reflectors or parabolic "mirrors,"fractal membranes, metamaterial microwave lenses, etc. Depending upon the Li-Baker detector design these optical microwave focusing devices can be positioned inside or outside of the GB.

Unlike the pure-inverse Gertsenshtein effect (G-effect), here under the synchro-resonance condition, coherence modulation of the HFGWs to the preexisting transverse photon flux of the Gaussian beam (GB) is predicted to produce the transverse (radial) first-order perturbative photon flux (PPF) or signal due to the presence of GWs as shown in Figure 1A, and the PPF has a maximum at a longitudinal symmetrical surface of the GB where the transverse background photon flux (BPF) or GB noise vanishes. Moreover, the PPF and the BPF have obviously different decay rates in the transverse direction, and the PPF reflected, for example by the fractal membranes, exhibits a very small decay to be compared with a very large decay of the much stronger BPF. Thus, such properties might provide a new way to distinguish the BPF (noise) and display the PPF (signal). The general form of the GB of a fundamental frequency mode is [44]

$$
\begin{aligned}
\psi= & \frac{\psi_{0}}{\sqrt{1+(z / f)^{2}}} \exp \left(-\frac{r^{2}}{W^{2}}\right) \\
& \exp \left(i\left[\left(k_{e} z-\omega_{e} t\right)-\tan ^{-1} \frac{z}{f}+\frac{k_{e} r^{2}}{2 R}+\delta\right]\right)
\end{aligned}
$$

where $r^{2}=x^{2}+y^{2}, k_{e}=2 \pi / \lambda_{e}, f=\pi W_{0}^{2} / \lambda_{e}$, $W=W_{0} \sqrt{1+(z / f)^{2}}, R=z+f^{2} / z, \quad \psi_{0}$ is the amplitude of the electric (or magnetic) field of the GB, $W_{0}$ is the minimum spot radius, $R$ is the curvature radius of the wave front of the GB. From Equation (A1) one finds $[1,39]$

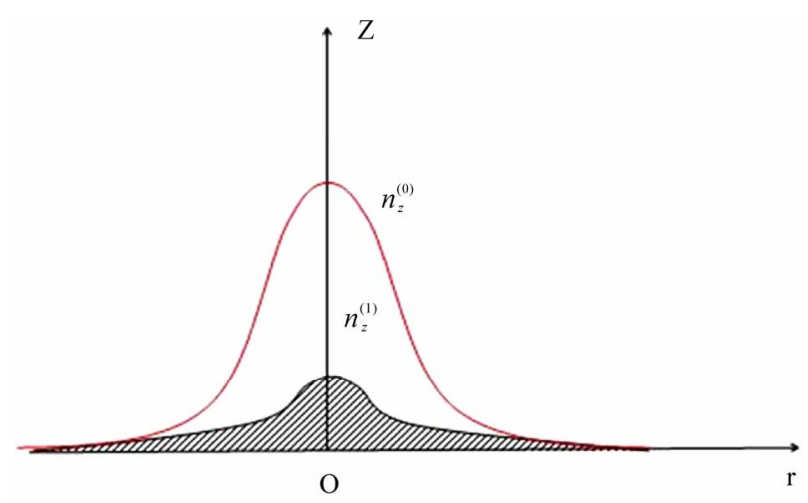

Figure 1A. First-order longitudinal PPF ( $n_{z}^{(1)}$ signal) and BPF ( $n_{z}^{(0)}$ noise) in the Li-Baker detector in the $\mathrm{z}$ direction as measured radially, $r$. 


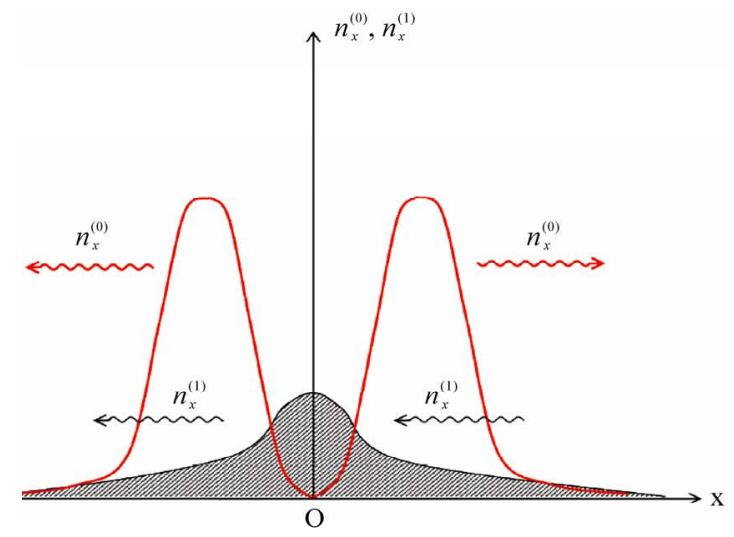

Region of $\mathrm{y}>0$

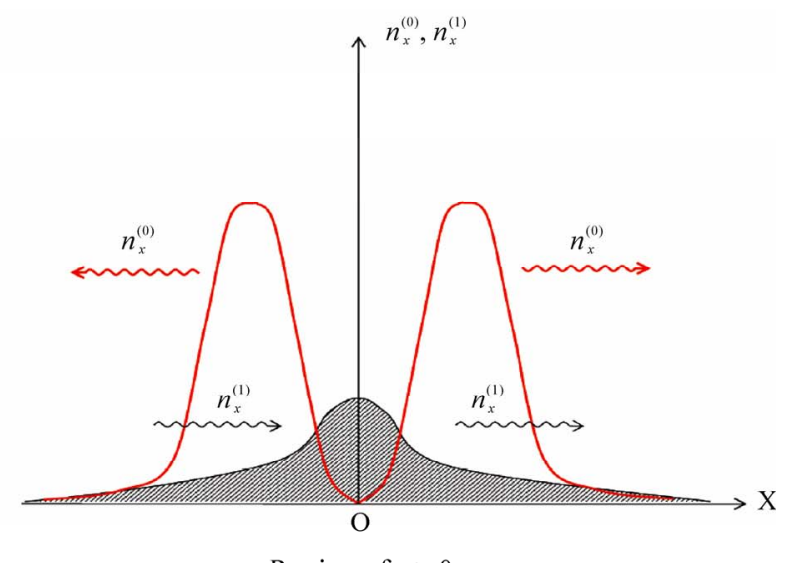

Region of $\mathrm{y}>0$

Figure 2A. Schematic diagram of strength distribution of the transverse BPF $n_{x}^{(0)}$ and PPF $n_{x}^{(1)}$ in the outgoing wave region of the GB [34,35].

$$
\begin{aligned}
& n_{x}^{(0)}=f_{x}^{(0)} \exp \left(-\frac{2 r^{2}}{W^{2}}\right), \\
& n_{y}^{(0)}=f_{y}^{(0)} \exp \left(-\frac{2 r^{2}}{W^{2}}\right), \\
& n_{z}^{(0)}=f_{z}^{(0)} \exp \left(-\frac{2 r^{2}}{W^{2}}\right)
\end{aligned}
$$

where $n_{x}^{(0)}, n_{y}^{(0)}, n_{z}^{(0)}$ represent the average values in the $\mathrm{x}-, \mathrm{y}-$ and $\mathrm{z}$ - directions of the BPF (noise) and $\left.f_{x}^{(0)}\right|_{x=0}=\left.f_{y}^{(0)}\right|_{y=0}=0,\left.\quad f_{z}^{(0)}\right|_{x=y=0}=\left.f_{z}^{(0)}\right|_{z \max }$. Because of the non-vanishing $n_{x}^{(0)}$ and $n_{y}^{(0)}$, the GB will be asymptotically spread as $|z|$ increases.

Unlike $n_{x}^{(0)}, n_{y}^{(0)}$ and $n_{z}^{(0)}$ (noise), the PPF $n_{x}^{(1)}$, $n_{y}^{(1)}$ and $n_{z}^{(1)}$ (signal) have different decay forms:

$$
\begin{aligned}
& n_{x}^{(1)}=f_{x}^{(1)} \exp \left(-\frac{r^{2}}{W^{2}}\right), \\
& n_{y}^{(1)}=f_{y}^{(1)} \exp \left(-\frac{r^{2}}{W^{2}}\right), \\
& n_{z}^{(1)}=f_{z}^{(1)} \exp \left(-\frac{r^{2}}{W^{2}}\right)
\end{aligned}
$$

where $f_{x}^{(1)}, f_{y}^{(1)}$ and $f_{z}^{(1)}$ are the functions of position $x, y, z$. Therefore, the decay rate of $n^{(1)}$ (signal) is slower than that of $n^{(0)}$ (noise).

In the Li-Baker detector the first-order longitudinal PPF $n_{z}^{(1)}$ and the BPF $n_{z}^{(0)}$ have the same propagating direction, and $n_{z}^{(0)}$ is much lager then $n_{z}^{(1)}$ in most of the nearby regions. Thus, $n_{z}^{(1)}$ will be swamped by the $n_{z}^{(0)}$ in such regions. However, the $n_{z}^{(1)}$ and $n_{z}^{(0)}$ will exhibit a comparable order of magnitude in the "far-axis region" ( $r>30 \mathrm{~cm}$ about the distance of six radii of the GB as shown in Figure 2A) due to the different decay

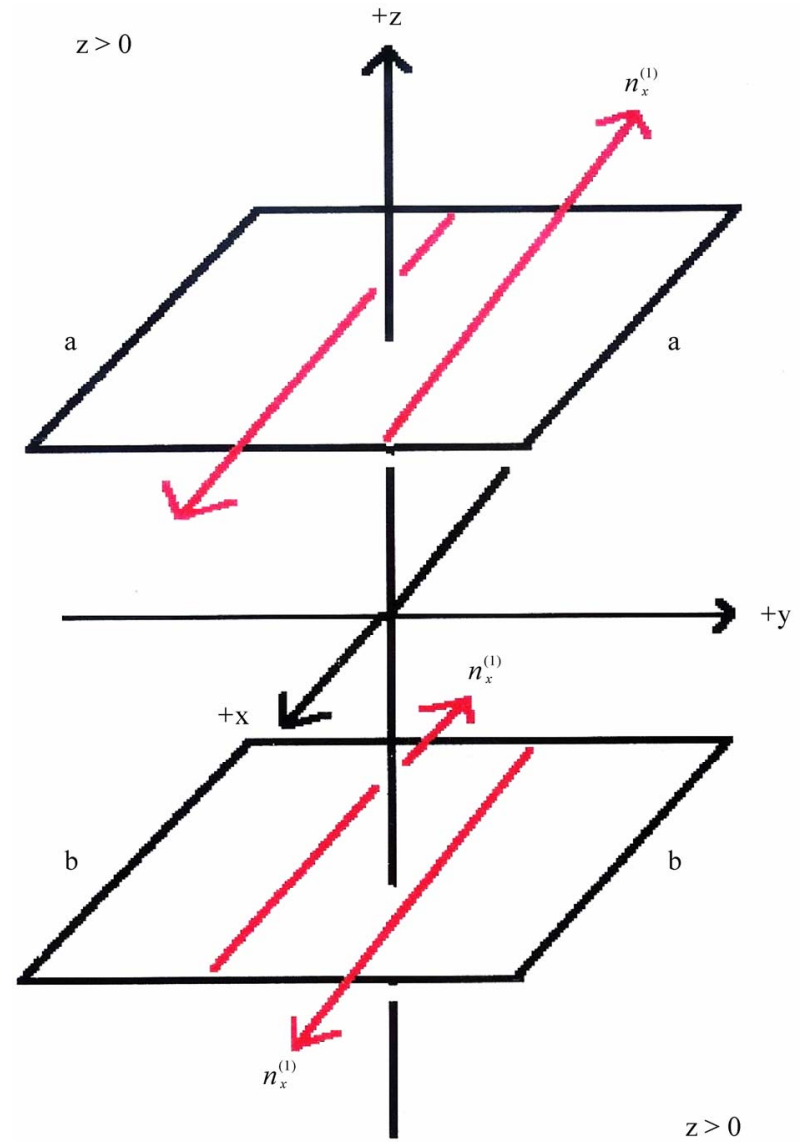

Figure 3A. The interaction of the GB, magnetic field and HFRGWs in the Li-effect produces $n_{x}^{(1)}$ photons.

rates and the $n_{z}^{(1)}$ will become larger than $n_{z}^{(0)}$ further out in the radial, $r$, direction. Therefore, as discussed in Section 5.8, the Li-Baker detector is photon-signal-limited, not quantum noise limited.

Unlike Figure 1A, here $\left.n_{x}^{(0)}\right|_{x=0}=0$ while 
ET $A L$.

$\left.n_{x}^{(1)}\right|_{x=0}=n_{x \max }^{(1)}$. Thus, $n_{x}^{(1)} \Delta t$ (accumulated signal) can be effectively larger than the background noise photon fluctuation $\left(n_{x t o t}^{(0)} \Delta t\right)^{1 / 2}$ at the yz-plane and at the parallel surfaces near the yz-plane, provided that the total noise photon flux passing through the surface can be effectively suppressed as discussed in Section 5. Because $n_{x}^{(1)}$ propagates along apposite directions in the regions of $y>0$ and $y<0$ in the GB, there is conservation of total momentum in the coherent resonance interaction and there is also an ability to focus half of the $n_{x}^{(1)}$, which are directed to the center of the GB, at the two microwave receivers at opposite ends of the $\mathrm{x}$-axis. The reverse mirror image of Figure 2A in the xy-plane $(\mathrm{z}<$ 0 ), shown in Figure 3A as b-b, insures that there is conservation of angular momentum and the differrentiation of the interaction volume into octants about the origin (center or intersection of the axes of the GB and the static magnetic field) is established by $[1,39]$.

\section{Acknowledgements}

The work on this Appendix is supported in part by the National Nature Foundation of China, grant No. 11075224. 